$$
\text { DOE/PC/ } / 92 / 20-T / 0
$$

\title{
Quarterly Report Final
}

\section{ADVANCED COAL LIQUEFACTION}

To

PITTSBURGH ENERGY TECHNOLOGY CENTER

April 1, 1995

to

June 30, 1995

DE-AC22-93PC92120

Media and Process Technology Inc. 1155 William Pitt Way

Pittsburgh, PA 15238
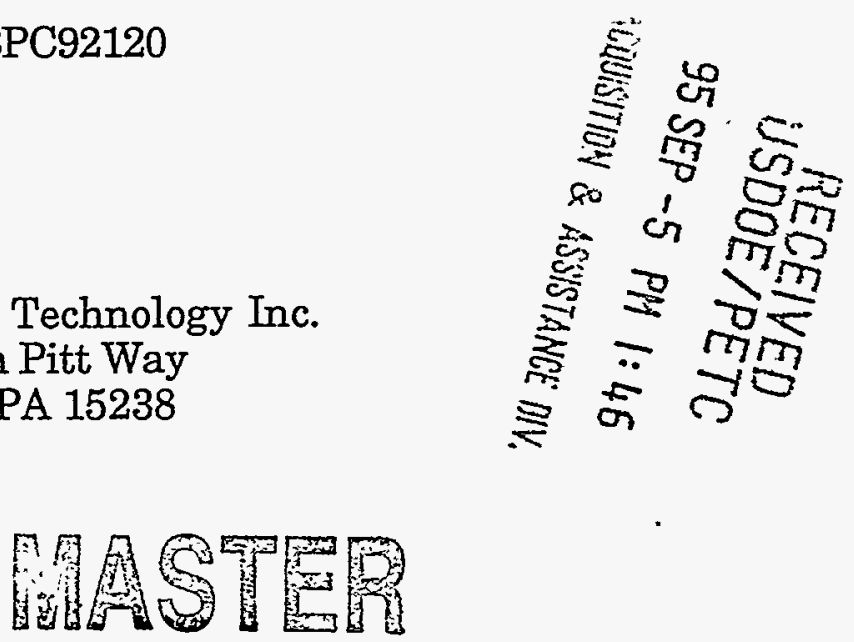

DISTRIBUTION OF THIS DOCUMENT IS UNLEATED 
Several permeation run was conducted in this quarter to prove the functionality of the experimental system. Compound \#9 in tetralin was decomposed to bibenzyl and methyl bibenzyl at $400^{\circ} \mathrm{C}$ and $200 \mathrm{psi}$. Since no carbon catalyst was used, the reaction was resulted from the surface of the ceramic membrane coated with carbon. Major reaction products were identified from the chromatogram. Although the permeate composition analysis fails to close the material balance, the experimental runs demonstrated the viability of the experimental system and technical concept. In the next quarter, we will continue the similar test to reach a satisfactory material balance.

\section{Discussion}

\section{Characterization of Ceramic Membrane}

A $10^{\prime}$ Si-CVD modified ceramic membrane was used for this quarter of study. This membrane was characterized with helium and nitrogen at room temperature and $600^{\circ} \mathrm{C}$ as shown in Table 1 . Based upon this characterization data, we believe that the pore size of this membrane is around $10 \AA$. It could perform some limited separation of compound \#9 from tetralin according to our previous separation experience using other membranes. This membrane was coated with carbon before the filtration study to minimize the possible fouling potential of the porous structure of the membrane. The gas permeation flux measured before and after carbon coating (shown in Table 1) indicates that pore size distribution was not altered noticeably through carbon coating.

\section{Permeation of Compound \#9/Tetralin}

The above membrane (Si519) was used to perform the liquid filtration at $400^{\circ} \mathrm{C}$ with 200 psi of pressure drop. The feed contains $0.38 \mathrm{wt} \%$ of compound \#9 (at retention time of 18.9) in tetralin. In addition, it contains a significant amount of an unknown compound (at retention time of 16), and other impurities came with tetralin. The compound \#9 in this feed was consumed as a result of the loss through permeation primarily. Since no other significant compounds were present in the feed at the end of the run (Figure A-15), it is believed that the decomposition of the compound \#9 at the surface of the membrane is insignificant. Also, the unknown compound behaves similarly to compound \#9, which could interfere the material balance calculation.

Figure 1 and Table 2 both summarize the concentration change throughout the permeation study which lasted 9 hours. Compound \#9 was reduced from 0.38 to 0.33 wt\%. Initially compound \#9 was collected along with its degradation products. However, after hour 3 , no compound \#9 was found in the permeate. Instead, bibenzyl was found throughout the entire permeation run with the concentration in the neighborhood of $0.05 \mathrm{wt} \%$. 0.2-0.3wt\% methyl bibenzyl concentration was found.

The permeation rate appeared constant throughout the entire run as shown in Figure 2. This constant flux was not observed in the past and could be possibly attributed to the larger pore size of the membrane. At the end of the run, black 
precipitate like carbon powder was collected in the permeate line. This indicates that some of the reactions may not be accounted for by the material increase and decrease shown in the chromatogram. Also, the combined concentration of bibenzyl and methyl bibenzyl appeared higher than that of compound \#9.

Evidently, the unknown peak was involved in the reaction process. Therefore, no material balance check was attempted. Additional study will be performed in the next quarter to determine the source of carbon and its impact on the material balance calculation. The unknown compound and its effect on the overall material balance will also be evaluated.

\section{DISCLAIMER}

This report was prepared as an account of work sponsored by an agency of the United States Government. Neither the United States Government nor any agency thereof, nor any of their employees, makes any warranty, express or implied, or assumes any legal liability or responsibility for the accuracy, completeness, or usefulness of any information, apparatus, product, or process disclosed, or represents that its use would not infringe privately owned rights. Reference herein to any specific commercial product, process, or service by trade name, trademark, manufacturer, or otherwise does not necessarily constitute or imply its endorsement, recommendation, or favoring by the United States Government or any agency thereof. The views and opinions of authors expressed herein do not necessarily state or reflect those of the United States Government or any agency thereof. 


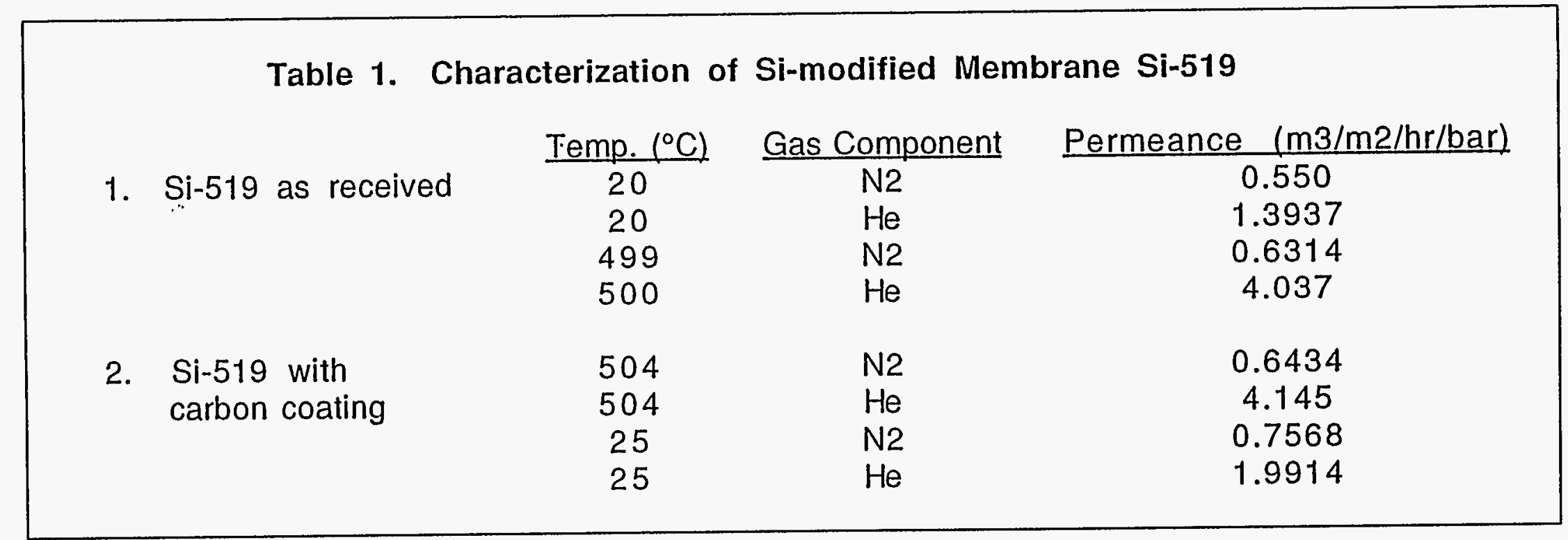


Table 2. Permeation of Coumpound \#9 in Tetralin through Si519 (7/20/95) at $400^{\circ} \mathrm{C}$ and $200 \mathrm{psi}$

\begin{tabular}{ccccc} 
Time (Hour) & Feed (Compound \#9) & Permeate (Compound \#9) & Permeate (Bibenzyl) & Permeate(Methyl Bibenzyl) \\
\cline { 2 - 4 } $1 .$. & 0.38 & 0.00 & 0.00 & 0.00 \\
2 & 0.37 & 0.07 & 0.10 & 0.31 \\
3 & 0.34 & 0.06 & 0.08 & 0.33 \\
4 & & 0.01 & 0.07 & 0.29 \\
5 & & 0.00 & 0.06 & 0.25 \\
6 & & 0.00 & 0.06 & 0.24 \\
7 & 0.34 & 0.00 & 0.05 & 0.24 \\
8 & & 0.00 & 0.05 & 0.22 \\
9 & 0.35 & 0.00 & 0.05 & 0.20 \\
0.33 & 0.00 & 0.05 & 0.19
\end{tabular}

Note: All concentrations in wt\% 


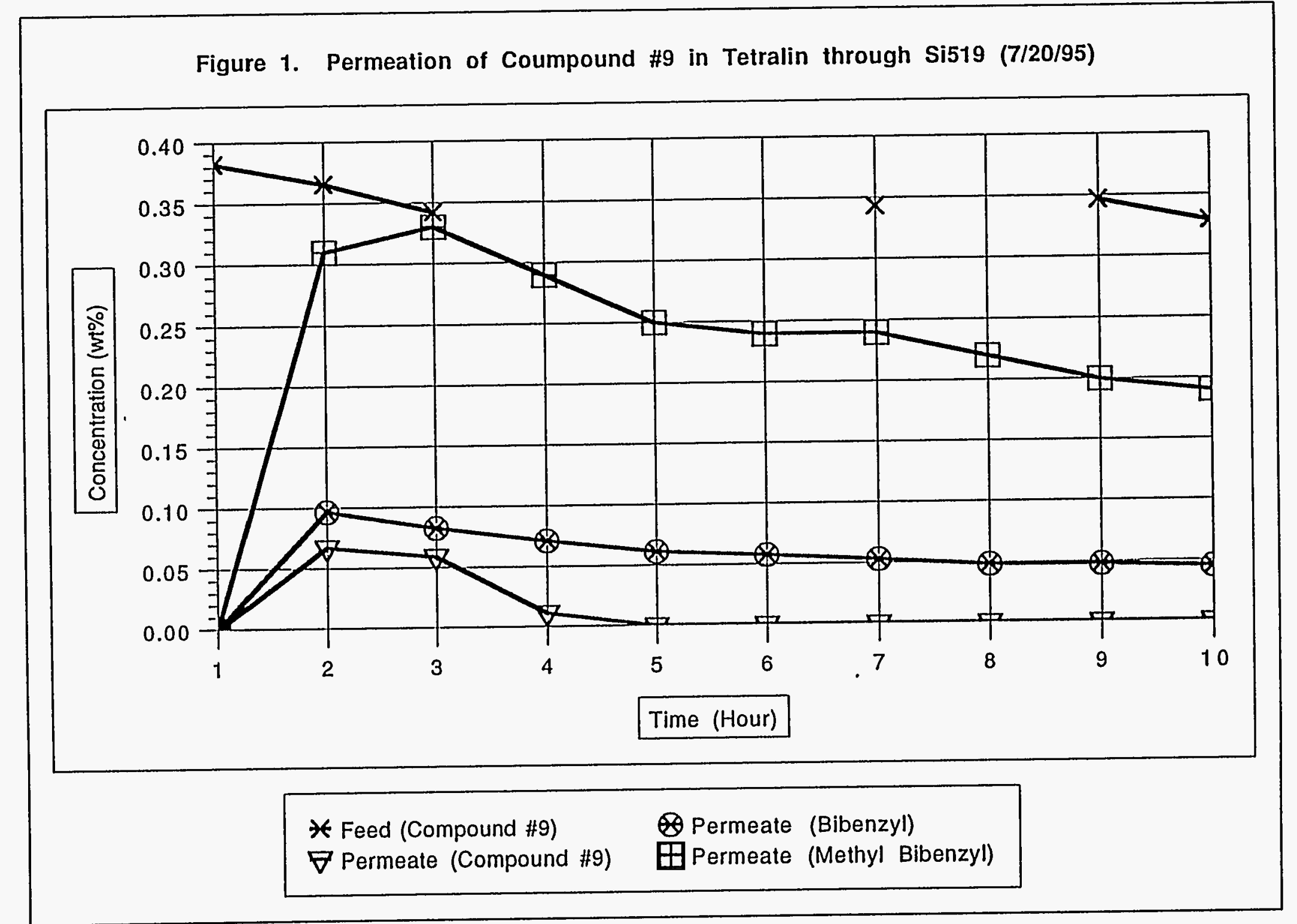


Figure 2. Permeation of Compound \#9 in Tetralin Through Si529 (7/20/95)

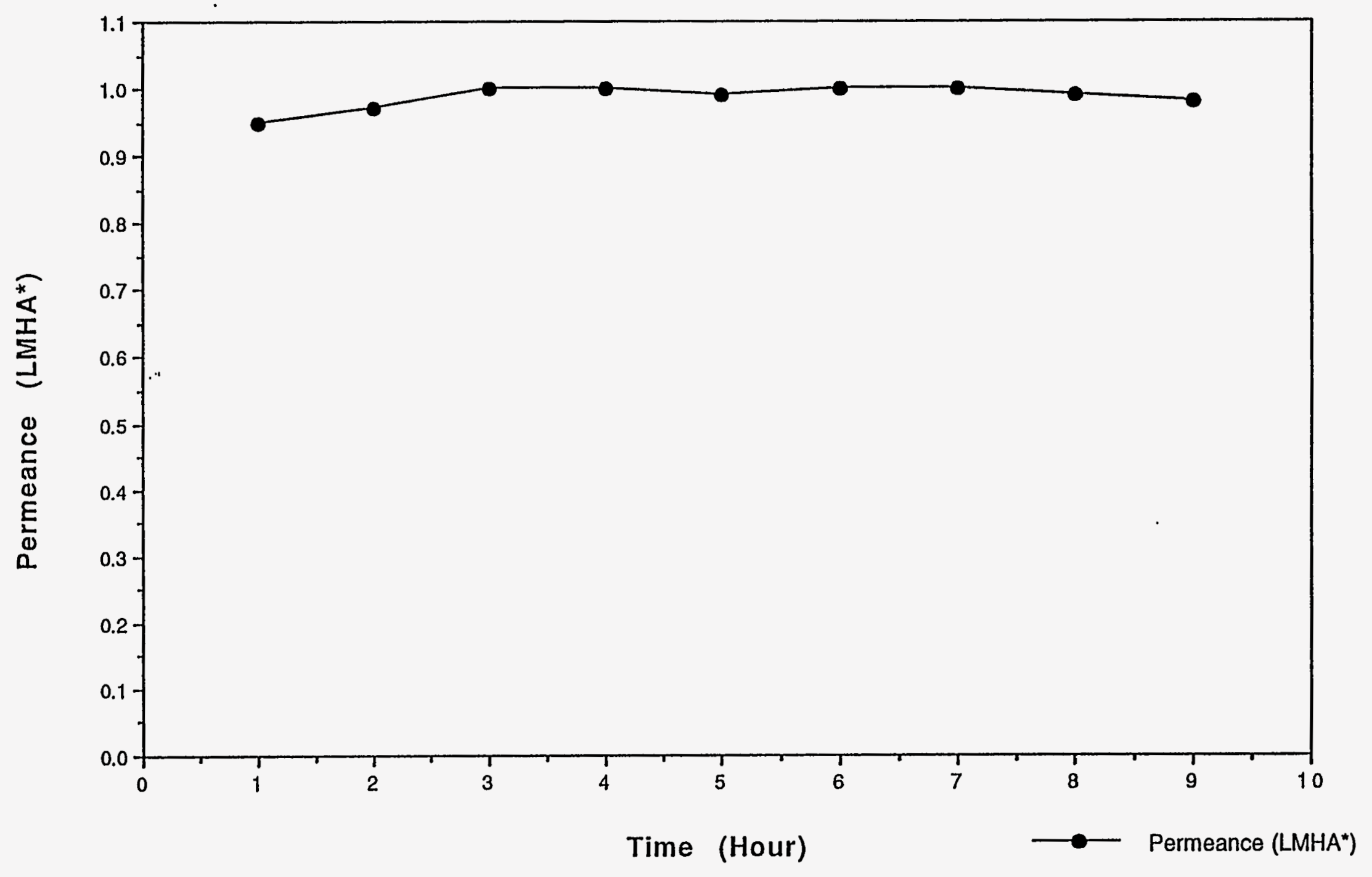

* Liter/m2/hr/bar 


\section{Appendix}

Figure A-1. Feed Before the Permeation Study

Figure A-2. Feed Corresponding to Hour 1 of Permeation Study

Figure A-3. Feed Corresponding to Hour 2 of Permeation Study

Figure A-4. Permeate Collected at Hour 1

Figure A-5. Permeate Collected at Hour 2

Figure A-6. Permeate Collected at Hour 3

Figure A-7. Permeate Collected at Hour 4

Figure A-8. Permeate Collected at Hour 5

Figure A-9. Feed Corresponding to Hour 6 of Permeation Study

Figure A-10. Permeate Collected at Hour 6

Figure A-11. Permeate Collected at Hour 7

Figure A-12. Feed Corresponding to Hour 8

Figure A-13. Permeate Collected at Hour 8

Figure A-14. Feed Corresponding to Hour 9

Figure A-15. Permeate Collected at Hour 9

Figure A-16. Feed Corresponding to the End of the Permeation Study 


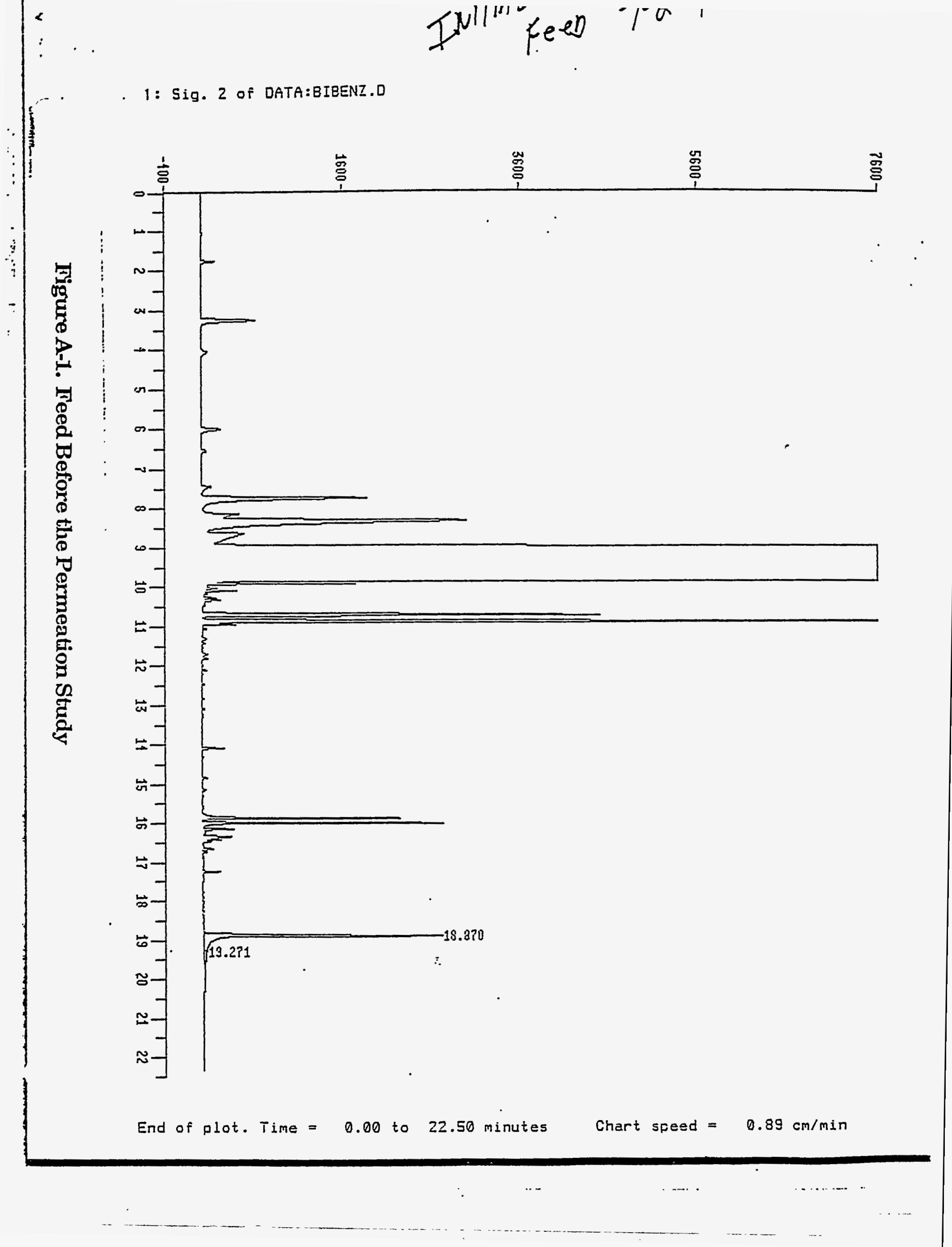


Operator:

Method File Name : WBBIBENZ.M

Sample Info:

Misc Info:

Integration File Name : OATA: GIBENZ.I

consisting of channels: 1 . GC Signal 2 . of BIBENZ.

Bottle Number : $\theta$ Repetition Number: 1

Calibration file: DATA:BIBENZ.Q Last Update: 19 Jul 95 10:02 am

Reference Peak Window: 5.00 Absolute Minutes

Non-Reference Peak Window: Q.10 Absolute Minutes

Sample fimount: 0.000 Uncalibrated Peak RF: 1.000 Muliipiier: 1.000

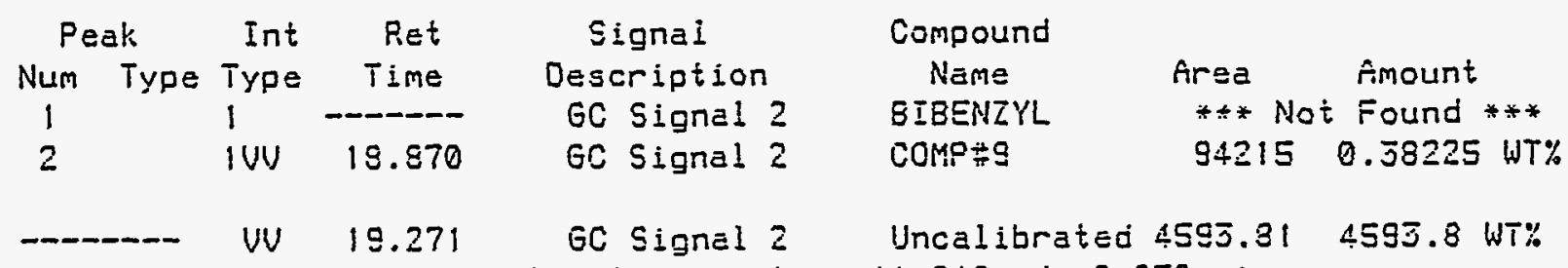

Sample paak \#1 not found at retention time $11.840 \div /-0.050 \mathrm{~min}$.

*** Not al! calibratid pesks found *** 


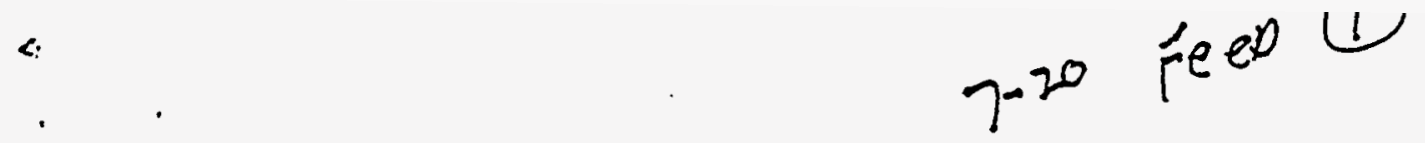

1: Sig. 2 of DATA:BIBENZ.D

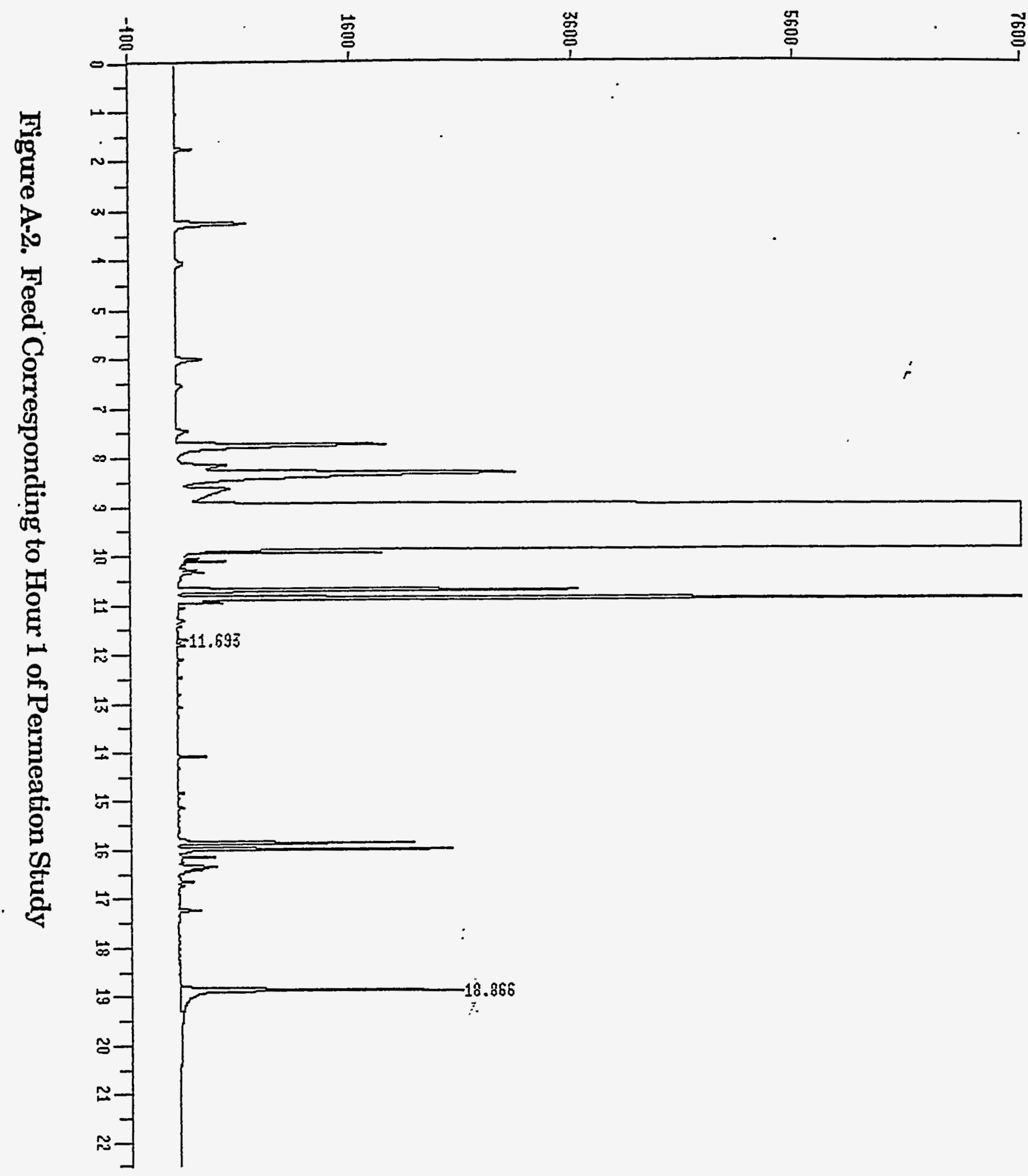

End of plot. Time $=0.00$ to 22.50 minutes Chert speed $=0.89 \mathrm{~cm} / \mathrm{min}$ 
Operator:

Method File Name : WBBIBENZ.M

Sample Info:

Misc Info:

Integration File Name : DATA:BIBENZ.I

consisting of channels : 1 . $6 C$ Signal 2

of BIBENZ.D

Bottle Number : 0 Repetition Number: 1

Calibration file: DATA:BIBENZ.Q Last Update: 19 Jul 95 10:02 am Reference Peak Window: 5.00 Absolute Minutes Non-Reference Peak Window: 0.10 Absolute Minutes

Sample Amount: 0.000 Uncalibrated Feak RF: 1.000 Multiplier: 1.000

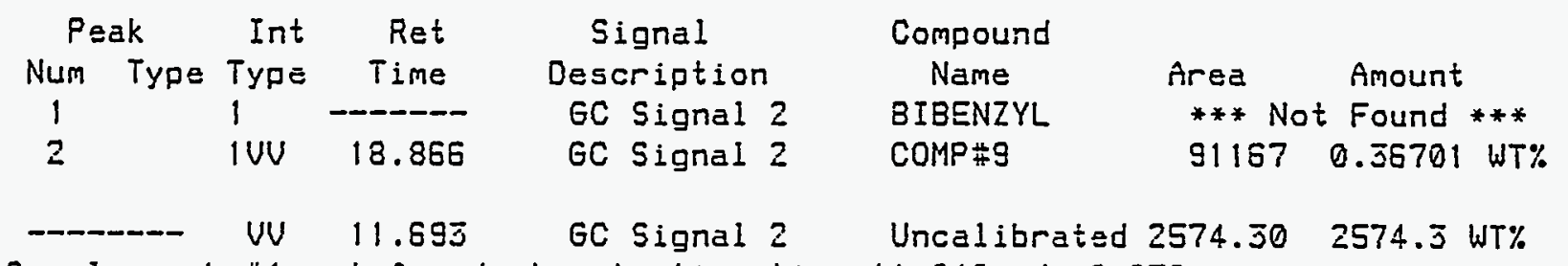

Sample peak \#1 not found at retention time $11.840+1-0.050 \mathrm{~min}$.

*** Not all calibrated peaks found *** 
$\left\{e^{20(2)}+2^{5}\right.$

$1: 5$ ig. 2 of DATA:BIBENZ.D

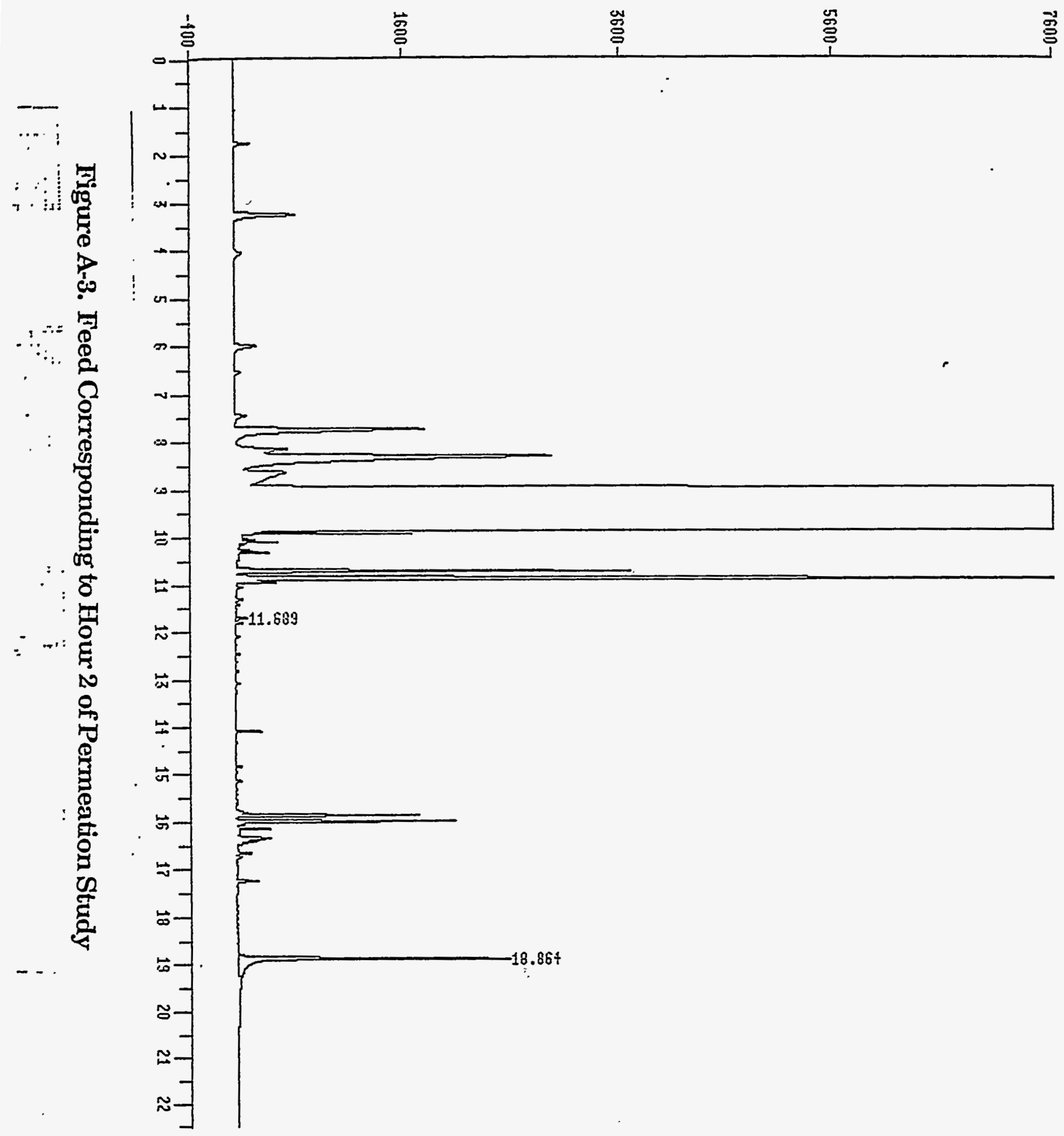

End of plot. Time $=0.00$ to 22.51 minutes Chart speed $=0.88 \mathrm{~cm} / \mathrm{min}$ 
Operator:

Method File Neme : WBbIBENZ.M

Sample Info:

Misc Info:

Integration File Name : DATA:EIBENZ.I

consisting of channels: 1 . GC Signal 2

of BIBENZ.D

Bottle Number : 0 Repetition Number: 1

Calibration file: DATA:BIBENZ.Q Last Update: 15 Jul 95 10:02 am

Reference Peak Window: 5.00 Absolute Minutes

Non-Reference Feak Window: 0.10 Absolute Minutes

Sample Amount: 0.000 Uncalibrated Peak RF: 1.000 Multiplier: 1.000

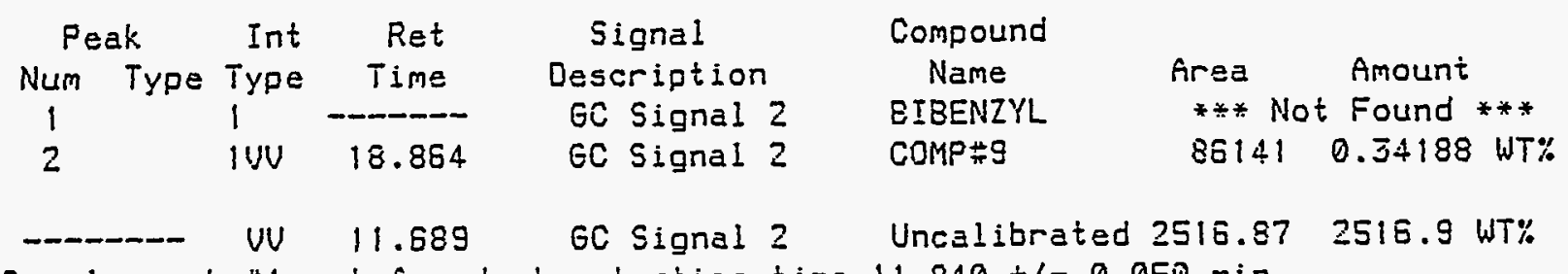

Sample peak $\# 1$ not found at retention time $11.840 \div 1-0.050 \mathrm{~min}$.

*** Not all celibrated peaks found *** 
(1) $\eta^{p e q m+20.95}$

1: Sig. 2 of DATA:BIEENZ.D

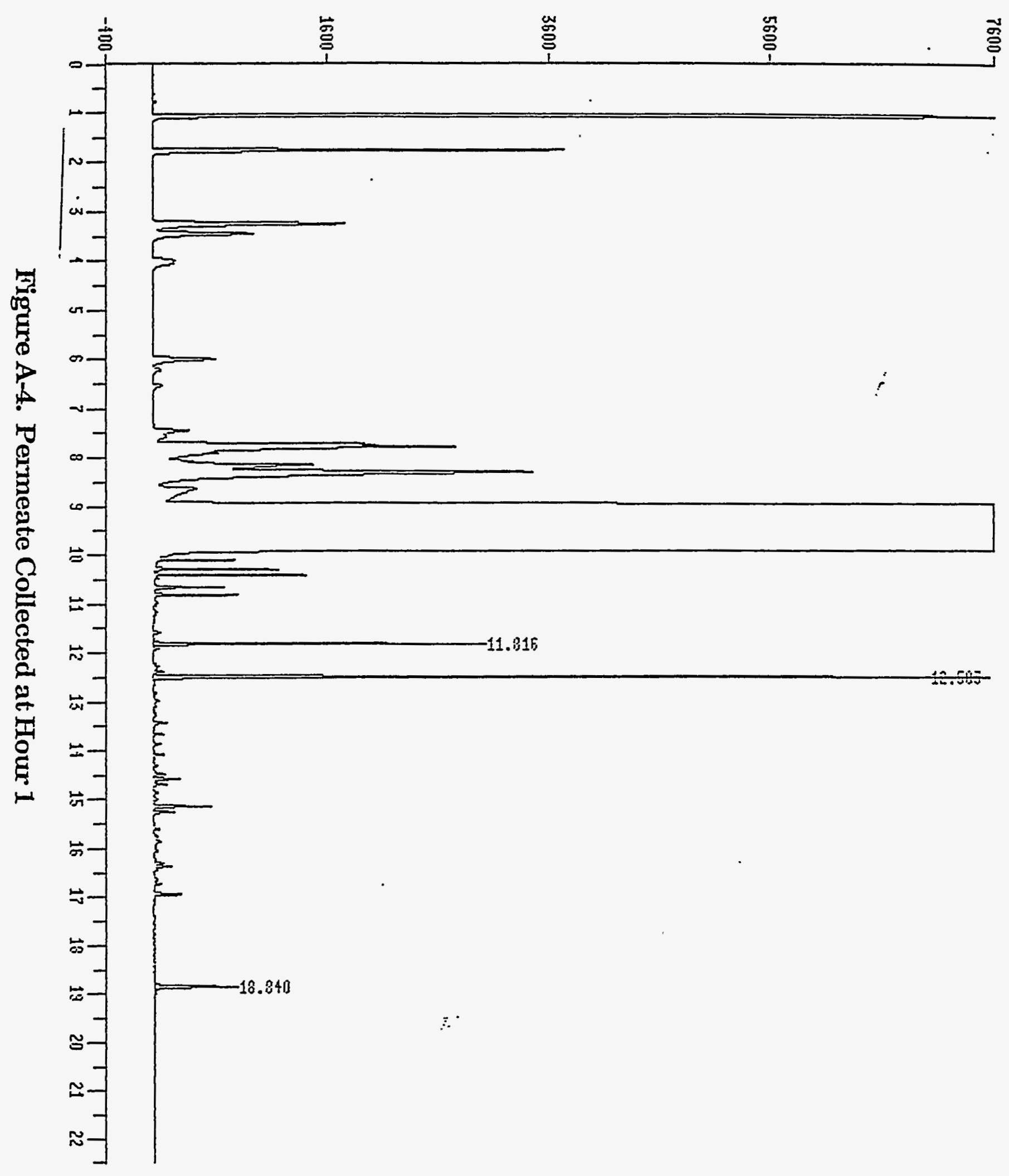

End of plot. Time $=0.00$ to 22.50 minutes Chart speed $=0.89 \mathrm{~cm} / \mathrm{min}$ 
Operator:

Method File Name : WBBIBENZ.M

Sample Info :

Mise Info:

Integration File Name : DATA:BIBENZ.I

consisting of channels : 1 . GC Signal 2 . of BIBENZ.0

Bottle Number : 0 Repetition Number: 1

Calibration file: DATA:BIBENZ.Q Last Update: 19 Jul 95 10:02 am

Reference Psak Window: 5.00 Absolute Minutes

Non-Feference Peak Window: 0.10 Absolute Minutes

Sampls Amount: 0.000 Uncalibrated Paak RF: 1.000 Multiplier: 1.000

\begin{tabular}{|c|c|c|c|c|c|c|c|}
\hline \multicolumn{2}{|c|}{ Peak } & Int & Ret & Signal & Compound & & \\
\hline Num & Type & $\begin{array}{l}\text { Type } \\
\text { IVU }\end{array}$ & $\begin{array}{c}\text { Time } \\
11.816\end{array}$ & $\begin{array}{l}\text { Description } \\
\text { GC Signal } 2\end{array}$ & $\begin{array}{l}\text { Name } \\
\text { BIBENLYL }\end{array}$ & $\begin{array}{l}\text { n̂rea } \\
44703\end{array}$ & $\begin{array}{l}\text { Amount } \\
0.082824 W T \%\end{array}$ \\
\hline 2 & & IUV & 18.838 & GC Signal 2 & COMP $\neq 9$ & 18017 & 0.058985 WT\% \\
\hline$-\infty$ & - & U & 12.504 & GC Signal 2 & Uncalibrated & 175416 & $175+16$ WT\% \\
\hline
\end{tabular}




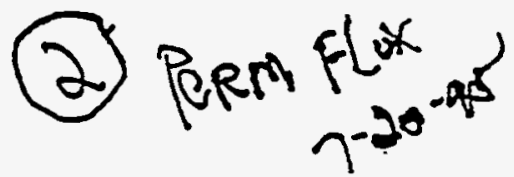

$1:$ Sig. 2 of DATA:BIBENZ.0

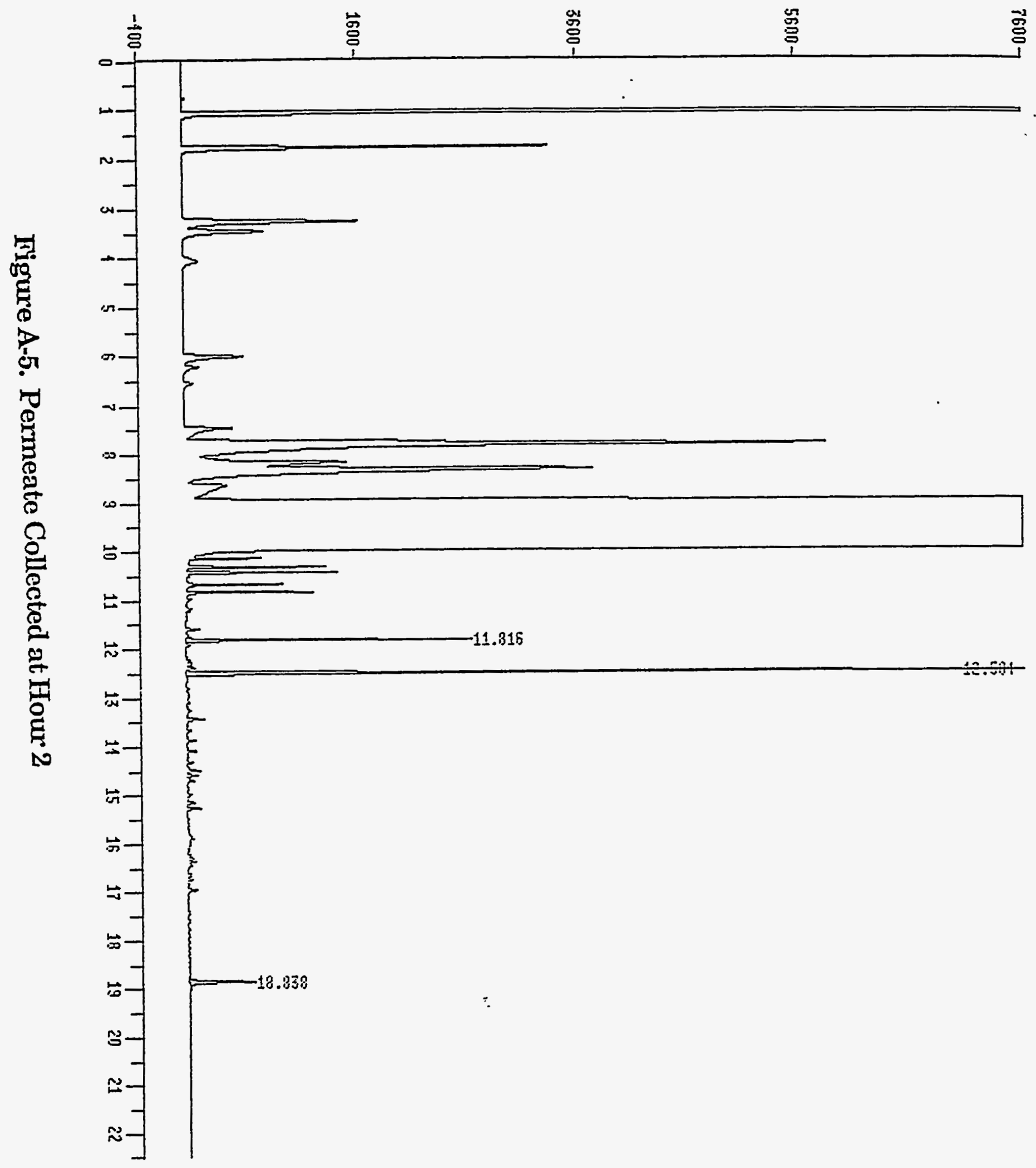

End of plot. Time $=0.00$ to 22.50 minutes Chart speed $=0.89 \mathrm{~cm} / \mathrm{min}$ 
Operator:

20 Ju1 $9511: 13 \mathrm{am}$

Method File Name : WBBIBENZ.M

Sample Info:

Misc Info:

Integration File Name : DATA:BIBENZ.I

consisting of channels : 1 . GC Signal 2

Of BIBENZ.D

Bottle Number : 0 Repetition Number: 1

Calibration file: DATA:BIEENZ.Q Lest Update: 19 jul 95 10:02 am

Reference Peak Window: 5.00 Absolute Minutes Non-Reference Peak Window: 0.10 Absolute Minutes

Sample Amount: 0.000 Uncalibrated Peak RF: 1.000 Multiplier: 1.000

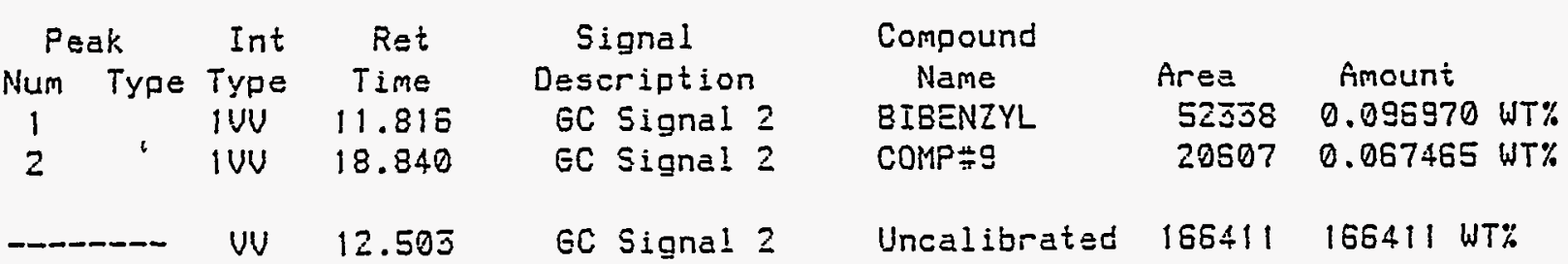


(3) $\operatorname{sen} n$

1: Sig. 2 of DATA:BIBENZ.D

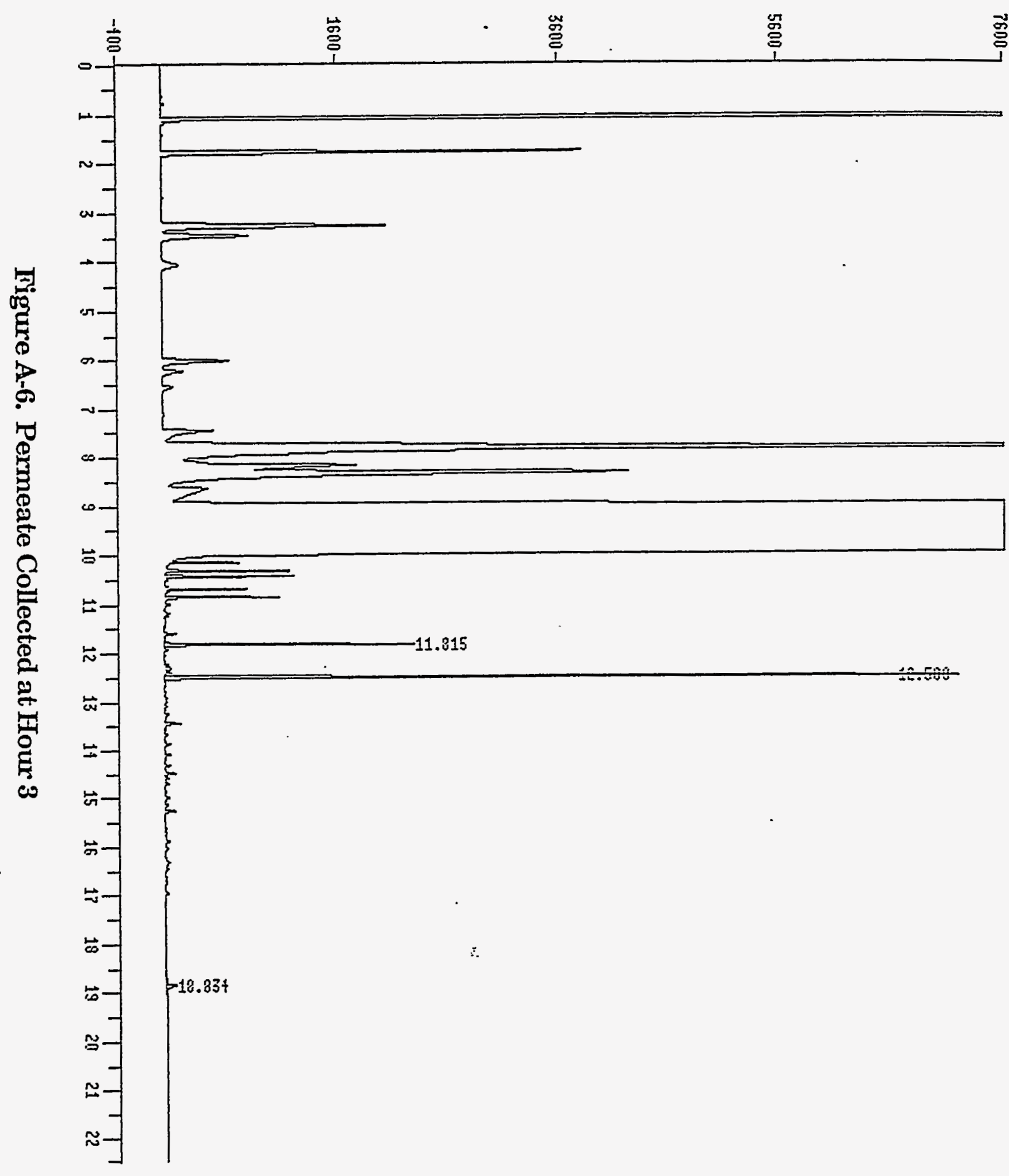

Fnd of mint. Time $=0.00$ to 22.51 minutes Chart speed $=0.88 \mathrm{~cm} / \mathrm{min}$. 
Operator:

Method File Name : WBBIBENZ.M

Sample Info:

Misc Info:

Integration Fil s Name : DATA:BIBENZ.I

consisting of channels: 1 . GC Signal 2

of BIBENZ.D

Bottle Number : 0 Repeitition Number: 1

Calibration file: DATA:BISENZ.Q Last Update: $19 \mathrm{Jul} 8510: 02 \mathrm{am}$

Reierence Peak Window: 5.00 Absolute Minutes

Non-Reference Peak Window: 0.10 Absolute Minutes

Sample Amount: 0.000 Uncalibrated Peak RF: 1.000 Multiplier: 1.000

\begin{tabular}{|c|c|c|c|c|c|c|c|}
\hline \multicolumn{2}{|c|}{ Peak. } & Int & $\operatorname{Ret}$ & Signal & Compound & & \\
\hline$\underset{1}{\text { Num }}$ & Type & $\begin{array}{l}\text { Type } \\
\text { IUU }\end{array}$ & $\begin{array}{c}\text { Time } \\
11.815\end{array}$ & $\begin{array}{l}\text { Description } \\
\text { GC Signal }\end{array}$ & $\begin{array}{c}\text { Name } \\
\text { BIBENZYL }\end{array}$ & $\begin{array}{l}\text { Area } \\
38530\end{array}$ & $\begin{array}{l}\text { Amount } \\
0.071586 \text { WT\% }\end{array}$ \\
\hline 2 & & 100 & 18.834 & EC Signal & COMP $\# Q$ & 3497.64 & 0.011451 WT\% \\
\hline & & uv & 12.500 & GC Signal & Uncalibrated & 154056 & 154056 WT\% \\
\hline
\end{tabular}




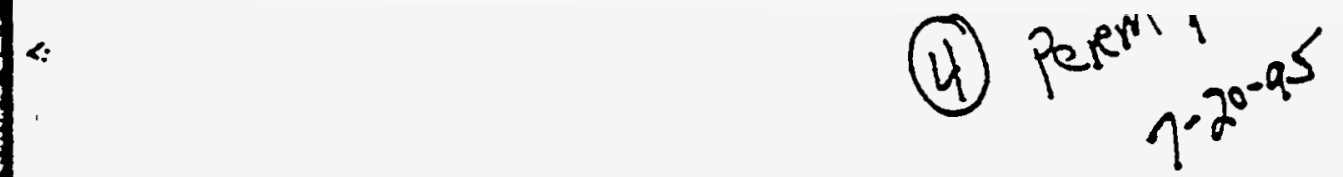

$1:$ Sig. 2 of DATA:BIBENZ.D

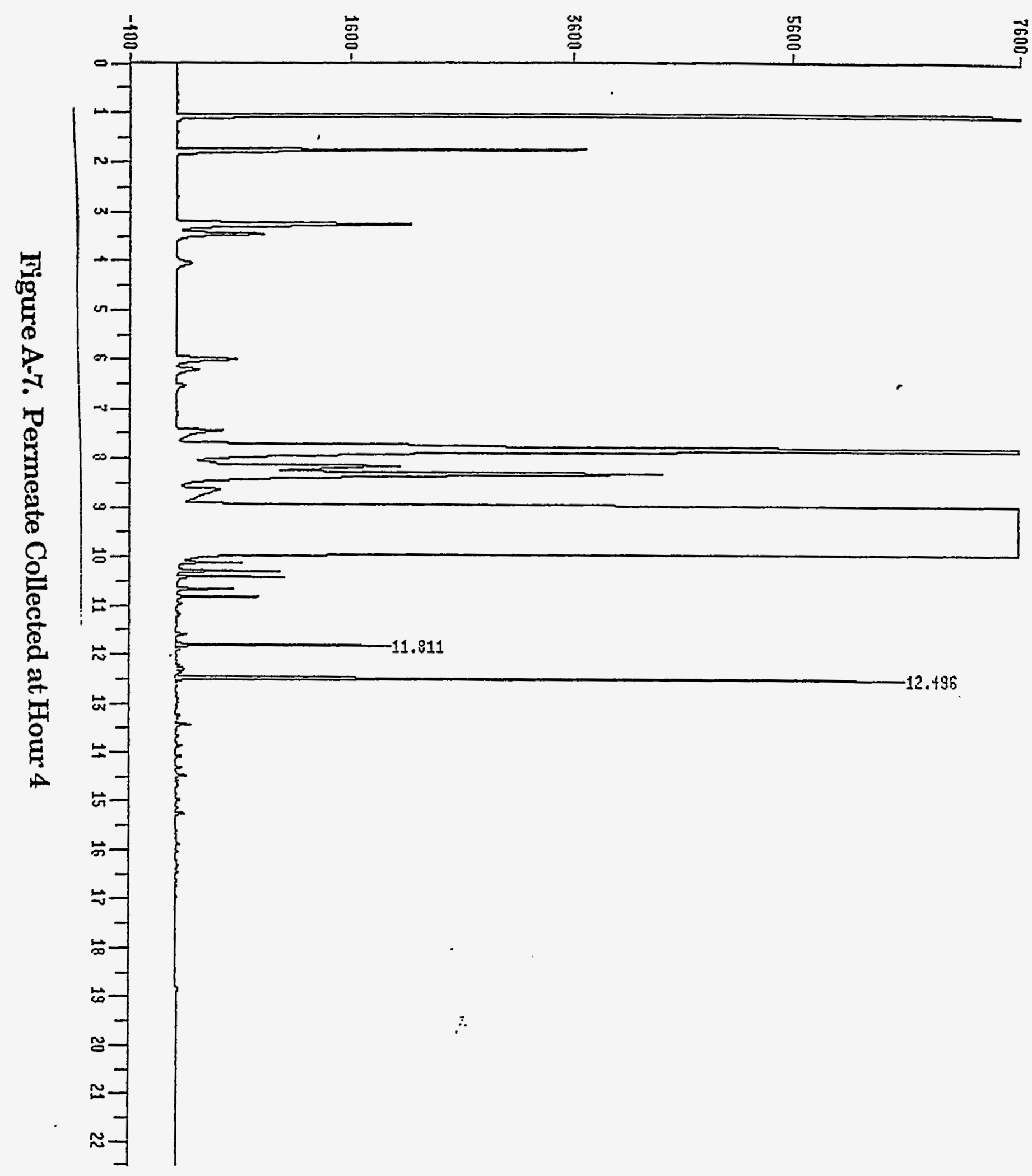

End of plot. Time $=0.00$ to 22.50 minutes Chart speed $=0.89 \mathrm{~cm} / \mathrm{min}$ 
Method File Name : WBBIBENZ.M

Sample Info:

Misc Info:

Integration File Name : DATA:BIBENZ.I consisting of channels : 1 . GC SignaI 2

Bottle Number : 0 Repetition Number: 1

of BIBENZ.D

Calibration file: DATA:BIBENZ.Q Last Update: 19 jul $9510: 02$ am Reference Peak Window: $\quad 5.00$ âbsolute Minutes Non-Reference Peak Window: 0.10 Absolute Minutes

Sample Amount: 0.000 Uncalibrated Peak RF: 1.000 Multiplier: 1.000

\begin{tabular}{|c|c|c|c|c|c|c|c|}
\hline \multicolumn{2}{|c|}{$P \approx a k$} & Int & Ret & Signal & Compound & & \\
\hline$\underset{1}{\text { Num }}$ & Type & $\begin{array}{l}\text { Type } \\
\text { IUV }\end{array}$ & $\begin{array}{l}\text { Time } \\
11.811\end{array}$ & $\begin{array}{l}\text { Qescription } \\
\text { 6C Signal } 2\end{array}$ & $\begin{array}{c}\text { Name } \\
\text { BIBENZYL }\end{array}$ & $\begin{array}{l}\text { Area } \\
33250\end{array}$ & $\begin{array}{l}\text { Amount } \\
0.061604 \text { WT\% }\end{array}$ \\
\hline 2 & & 1 & - - - - & GC Signal 2 & COMP $\div 9$ & $* * * \mathrm{No}$ & 6 Found **+ \\
\hline
\end{tabular}

- UU 12.496 GC Signal 2 Uncalibrated 134265154255 WT\% Sample peak \#2 not found at retention time $18.869+1-0.050 \mathrm{~min}$. ** Not all calibrated peaks found 


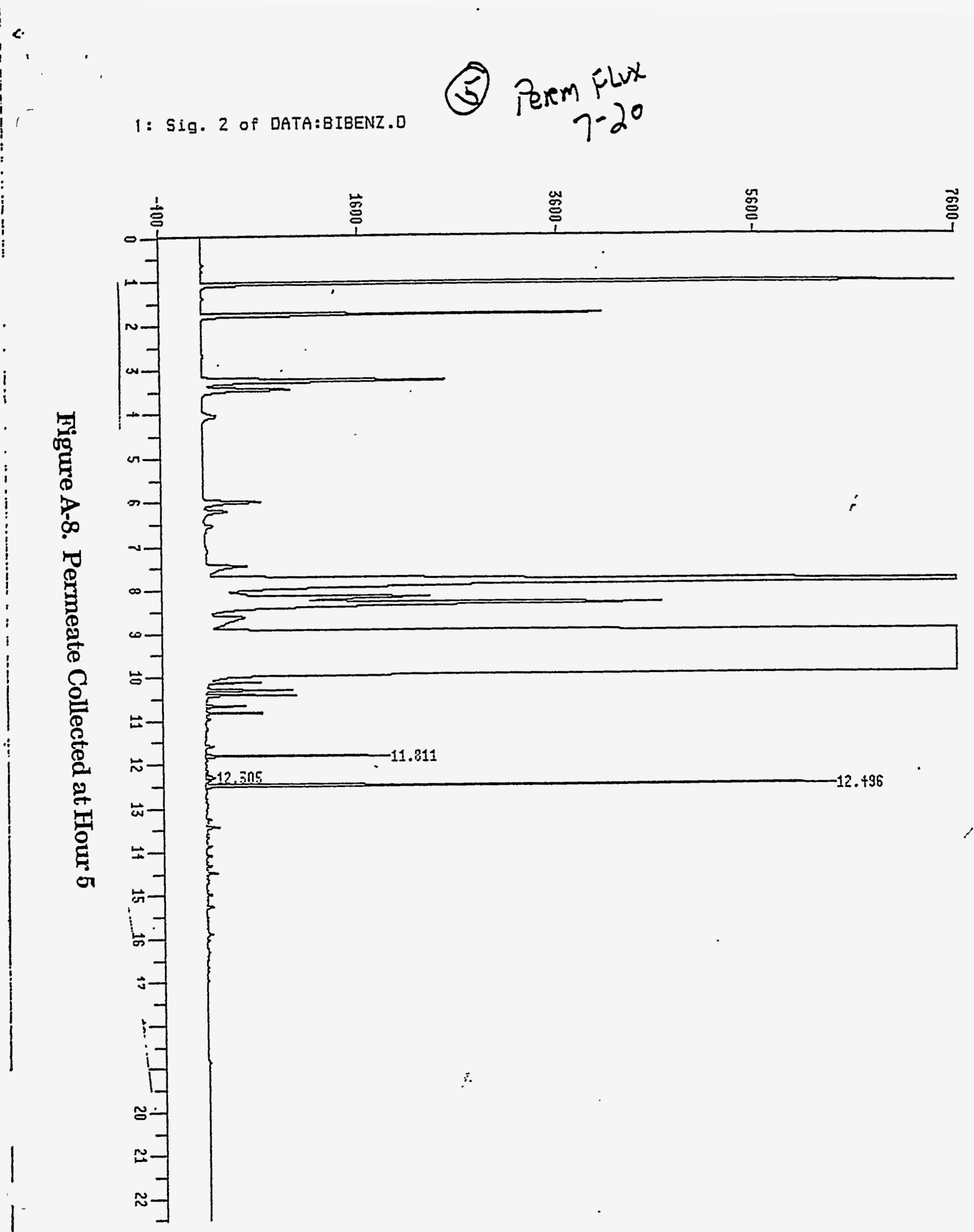

End of plot. Time $=0.00$ to 22.51 minutes Chart speed $=0.88 \mathrm{~cm} / \mathrm{min}$ 
Operator:

Method File Name : WBBIBENZ.M

$20 \mathrm{Jul} 95 \quad 3: 04 \mathrm{pm}$

Sample Info:

Misc Info:

Integration File Name : DATA:BIBENZ.I

consisting of channels : 1 . GC Signal 2 of BIBENZ.D

Bottle Number : $\theta$ Repetition Number: 1

Calibration file: DATA:BIBENZ.Q Last Update: $19 \mathrm{Jul} 95$ 10:02 am

$$
\text { Reference Peak Window: } 5.00 \text { Absolute Minutes }
$$

Non-Reference Peak Window: 0.10 Absolute Minutes

Sample Amount: 0.000 Uncalibrated Peak RF: 1.000 Multiplier: 1.000

\begin{tabular}{|c|c|c|c|c|c|c|}
\hline \multicolumn{2}{|c|}{ Peak } & Int & $R \in t$ & Signal & Compound & \\
\hline Num & Type & $\begin{array}{l}\text { Type } \\
\text { IUU }\end{array}$ & $\begin{array}{l}\text { Time } \\
11.811\end{array}$ & $\begin{array}{l}\text { Description } \\
\text { GC Signal } 2\end{array}$ & $\begin{array}{l}\text { Name } \\
\text { BIBENZYL }\end{array}$ & $\begin{array}{cc}\text { Area } & \text { fimount } \\
31291 & 0.057976 \text { WT\% }\end{array}$ \\
\hline 2 & & 1 & $-\infty-\infty$ & GC Signal 2 & COMP $\# 9$ & $* *$ Not Found $* * *$ \\
\hline
\end{tabular}

- U $12.305 \quad 6 C$ Signal $2 \quad$ Uncalibrated $2837.81 \quad 2837.8$ WT\%

-.-.- UU 12.456 GC Signal 2 Uncalibrated $130981 \quad 130981$ WT\%

Sample peak \#2 not found at retention time $18.889 \div 1-0.050 \mathrm{~min}$.

** Not all calibrated peaks found *** 
: $\quad$ : SIg. 2 of DATA:BIBENZ.D

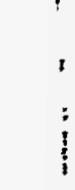

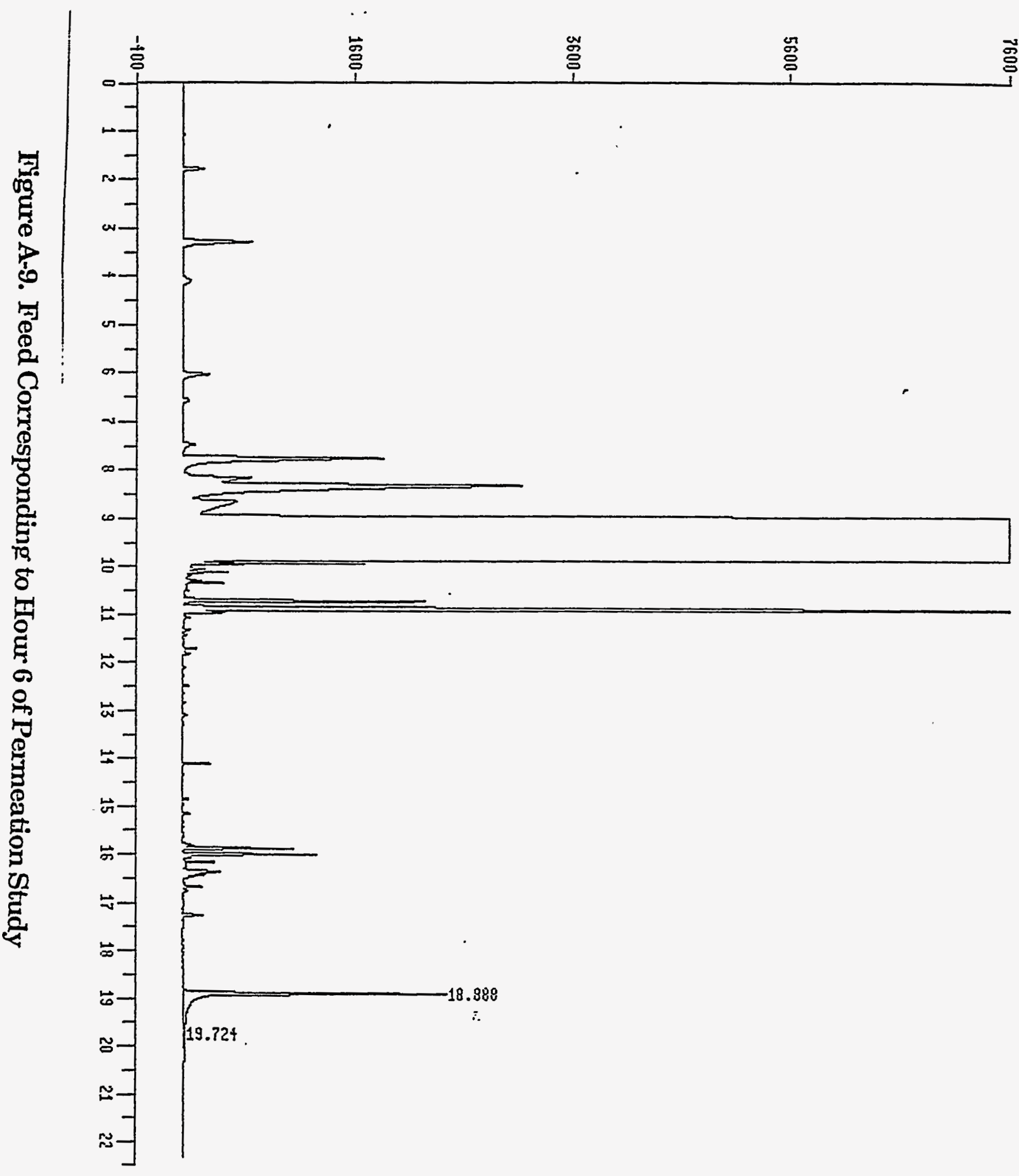

End of plot. Time $=0.00$ to $22.50 \mathrm{minutes}$ Chart speed $=0.8 \mathrm{~cm} / \mathrm{min}$ 
Operator:

Method File Name : WBBIBENZ.M

Sample Info:

Misc Info:

Integration File Name : DATA:BIBENZ.I

consisting of channels : 1 . GC Signal 2 . of BIBENZ.D

Bottle Number : 0 Repetition Number: $i$
21 JuI 95 11:06 am

Calibration file: DATA:GIBENZ.Q Last Update: 19 Jul $9510: 02$ am

Reference Peak Window: 5.00 Absolute Minutes

Non-Reference Peak Window: 0.10 Absolute Minutes

Sample Amount: 0.000 Uncalibrated Peak RF: 1.000 Multiplier: 1.000

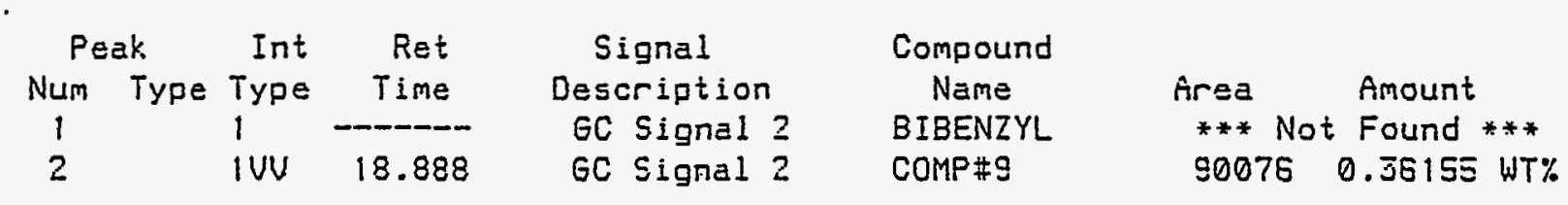

- UU 19.724 GC Signel 2 Uncelibrated $2722.07 \quad 2722.1$ WT\%

Sample peak \#1 not found at retention time $11.840+1-0.050 \mathrm{~min}$.

** Not all cailbrated peaks found *** 


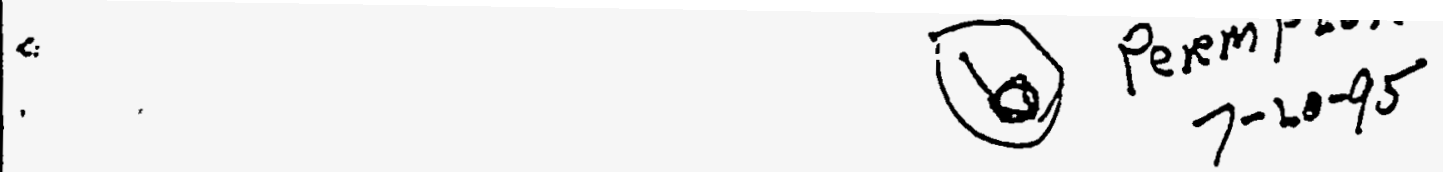

1: Sig. 2 of DATA:BIBENZ.D

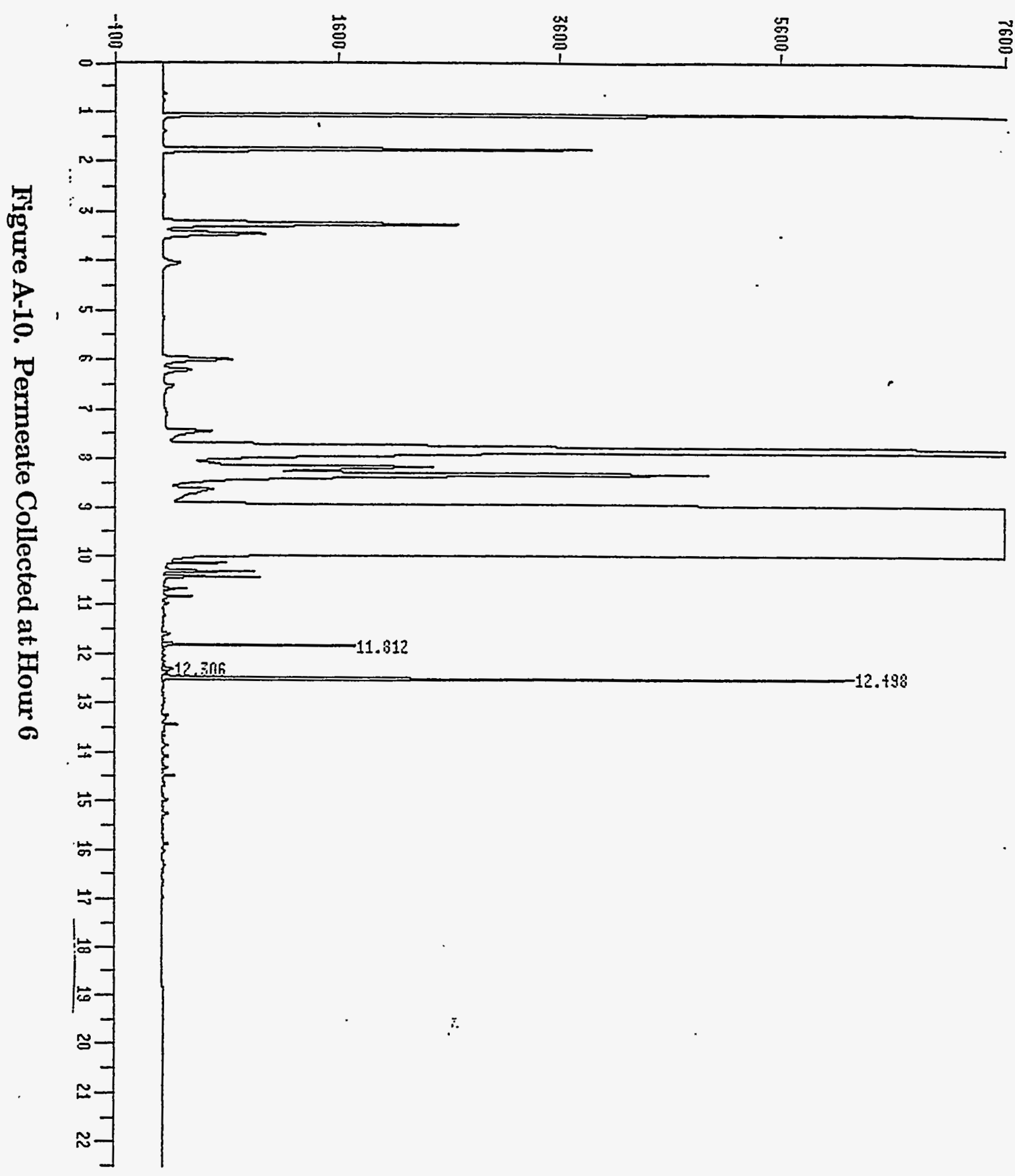

End of plot. Time $=0.00$ to 22.52 minutes Chart speed $=0.88 \mathrm{~cm} / \mathrm{min}$ 
Method File Name : WBEIBENZ.M

Sample Info:

Misc Info:

Integration File Name : DATA:BIBENZ.I

consisting of channels : 1 . GC Signal 2 of BIBENZ.D

Bottle Number : 0 Repetition Number: 1 .

Calibration file: DATA:BIBENZ.Q Last Update: 19 Jul 95 10:02 am

Reference Peak Window: $\mathbf{5 . 0 0}$ Absolute Minutes

Non-Reference Peak Window: 0.10 Absolute Minutes

Sample Amount: 0.000 Uncalibrated Peak RF: 1.000 Multiplier: 1.000

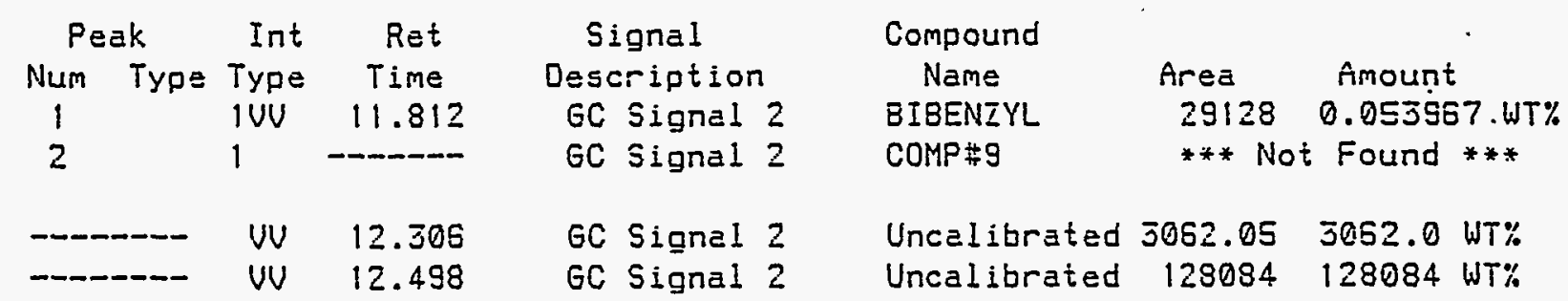

Sample peak $\# 2$ not found at retention time $18.869+1-0.050 \mathrm{~min}$.

*** Not all calibrated peaks found *** 
*. (7) Perm 1.2095

$1:$ Sig. 2 of DATA:BIBENZ.D

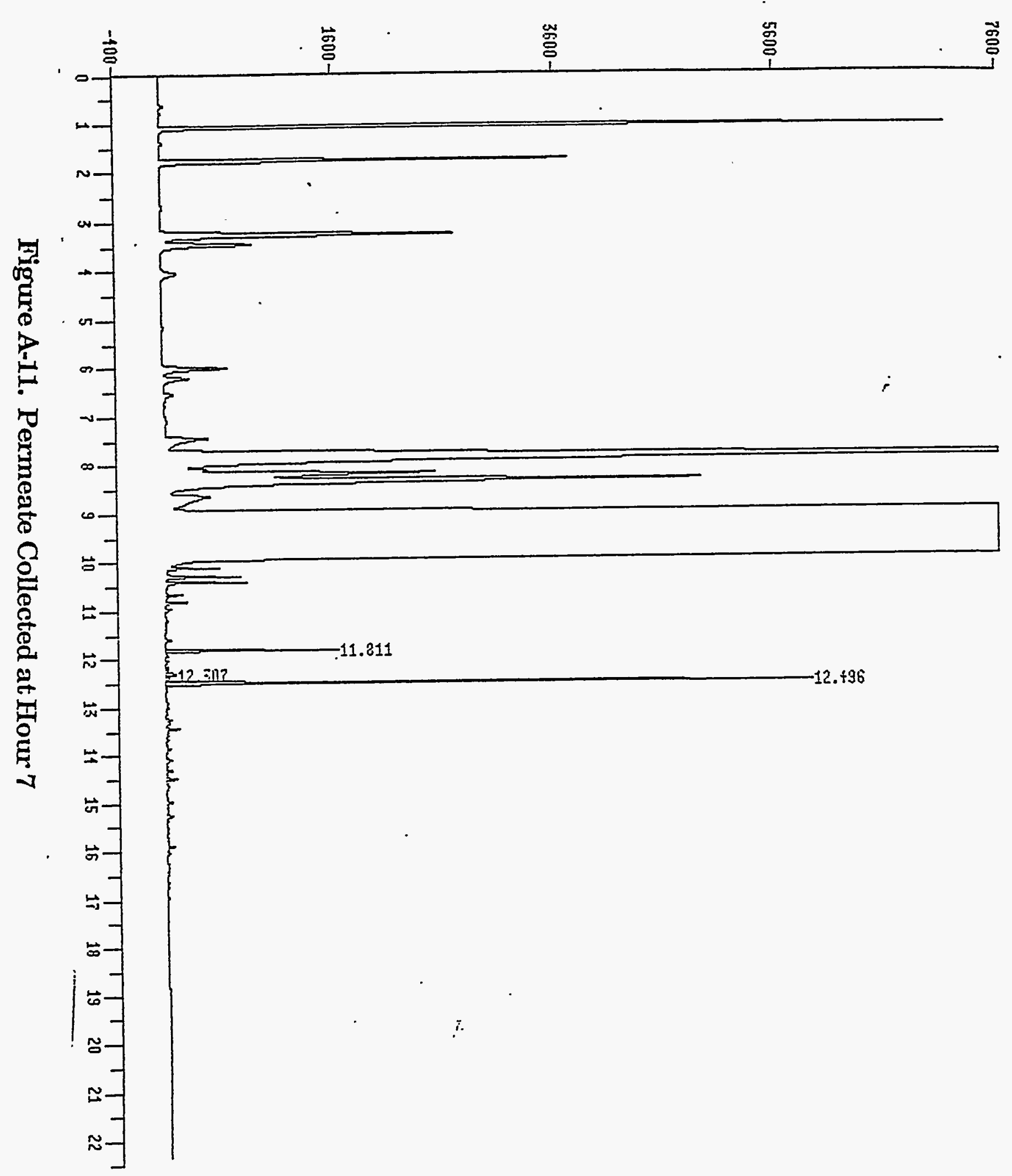

End of plot. Time $=0.00$ to 22.50 minutes Chart speed $=0.89 \mathrm{~cm} / \mathrm{min}$ 
Operator:

Method File Name : WBBIBENZ.M

Sample Info:

Misc Info:

Integration File Name : DATA:BIBENZ.I

consisting of channels : 1 . GC Signal 2 of BIBENZ.D

Botile Number : 0 Repetition Number: 1

Calibration file: DATA: BIBENZ.Q Last Update: 19 Jul 95 10:02 am

Reierence Peak Window: 5.00 fbsolute Minutes

Non-Reference Peak. Window: 0.10 Absolute Minutes

Sample fmount: 0.000 Uncalibrated Peak RF: 1.000 Multiplier: 1.000

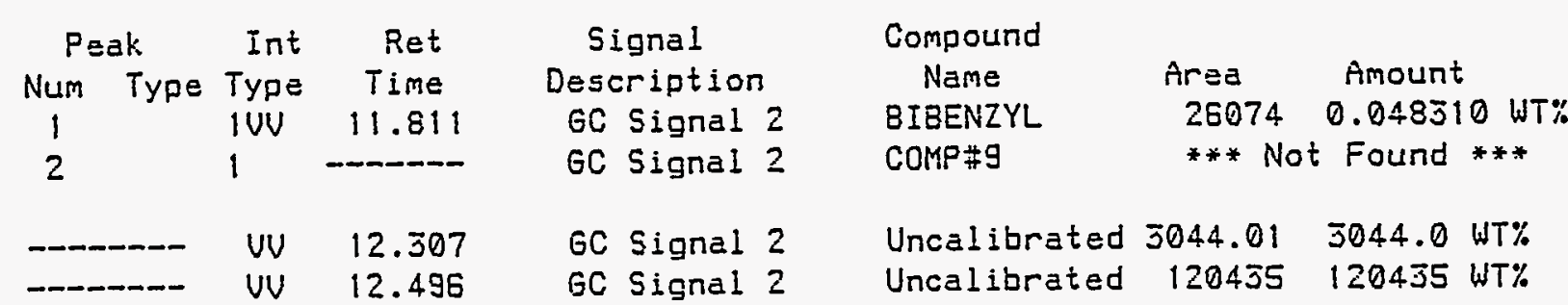

sample peak $\# 2$ not found at retention time $18.869+1-0.050 \mathrm{~min}$.

** Not all calibrated peaks found *** 


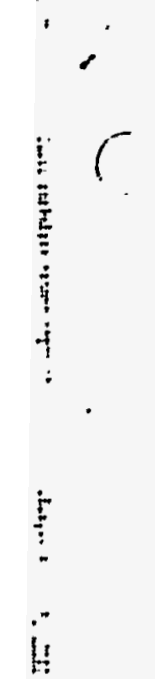

1: Sig. 2 of DATA:BIBENZ.D

$$
\text { fee: } 8
$$

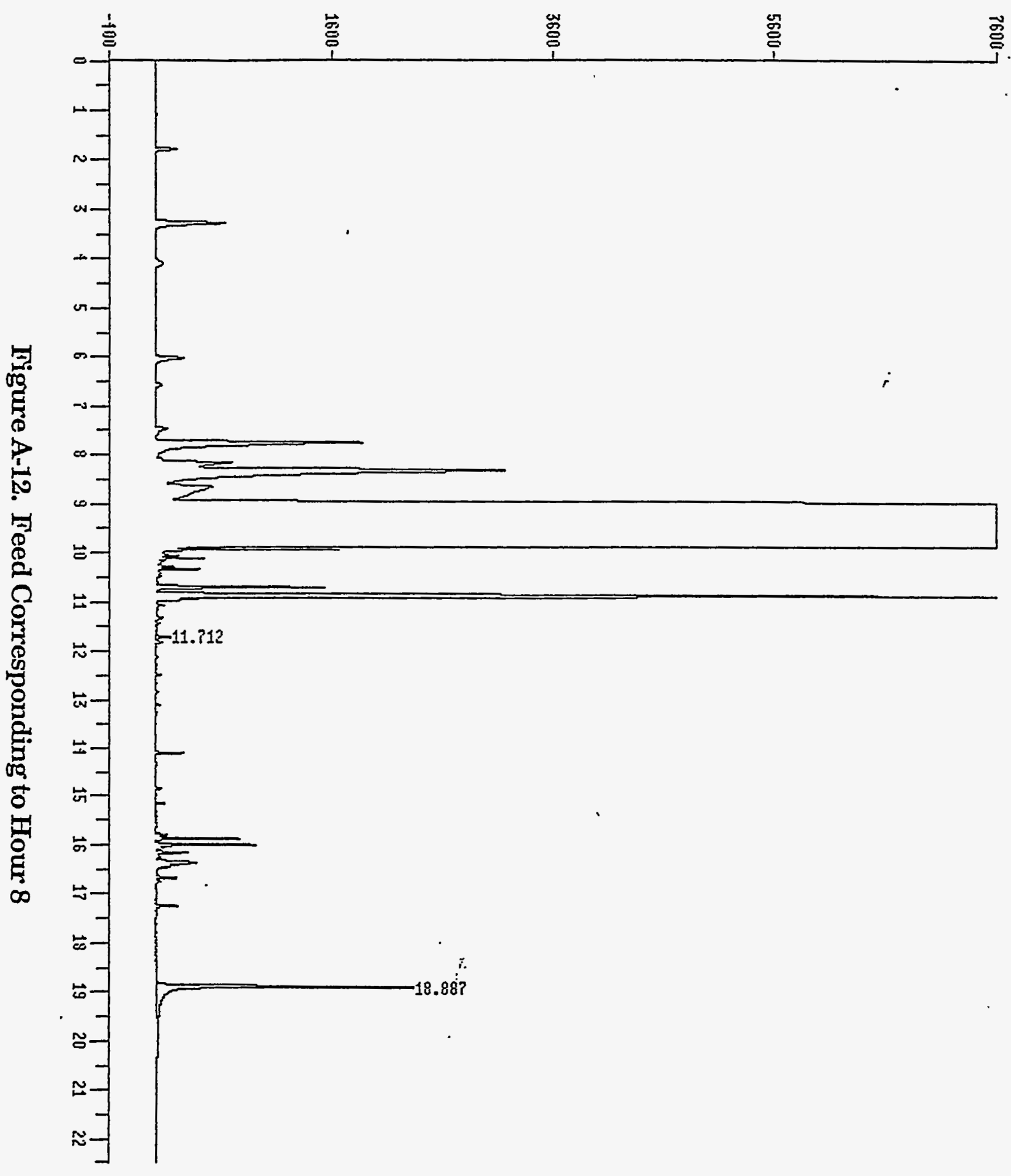

End of plot. Time $=$ 
Operator:

21 Jul 95 12:14 pm

Method File Name : WBBIBENZ.M

Sample Info:

Mise Info:

Integration File Name : DATA:BIBENZ.I

consisting of channels : 1 . GC Signal 2 ' of BIBENZ.D

Bottle Number : 0 Repetition Number: 1
Calibration file: DATA:PIBENZ.Q Last Update: 19 Jul 95 10:02 am Reference Peak Window: 5.00 Absolute Minutes Non-Reference Peak Window: 0.10 Absolute Minutes

Sample Amount: 0.000 Uncalibrated Peak RF: 1.000 Multiplier: 1.000

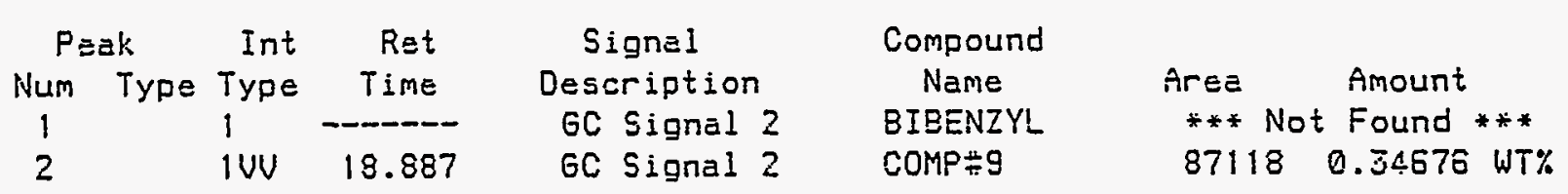

Wample peak $\# 1$ not found at retention time $11.840+1-0.050 \mathrm{~min}$.
-** Not all calibrated peaks found $* * *$ 
Operator:

Method File Name : WBBIBENZ.M

Sample Info:

Misc Info:

Integration File Name : DATA:BIBENZ.I

consisting of chennels : 1 . GC Signal 2

Bottle Number : 0 Repetition Number: 1

of BISENZ.0

Calibration file: DATA:BIBENZ.Q Last Update: 19 Jul 95 10:02 am

Reference Peak Window: 5.00 fbsolute Minutes

Non-Reference Peak Window: 0.10 Absolute Minutes

Sample Amount: 0.000 Uncalibrated Peak RF: 1.000 Multiplier: 1.000

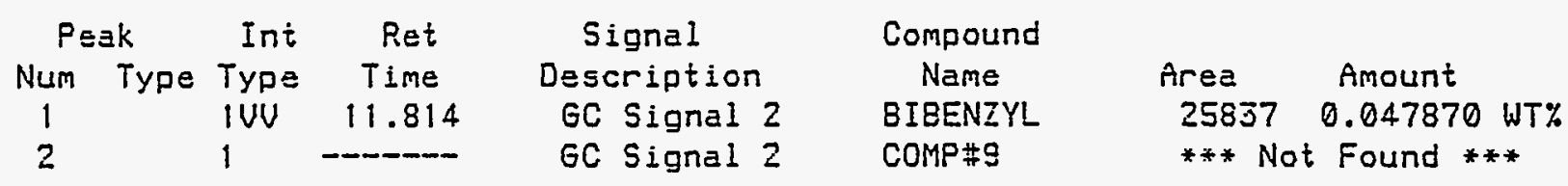

$\begin{array}{llllll}\text { UU } & 12.308 & \text { GC Signal } 2 & \text { Uncalibrated } 3770.85 & 3770.8 \text { WT\% }\end{array}$

Sample peak \#2 not found at retention time $18.869 \div 1-0.050 \mathrm{~min}$.

*** Not all calibrated peaks found *** 
(a) tery

1: Sig. 2 of DATA:BIBENZ.D

$n-20$

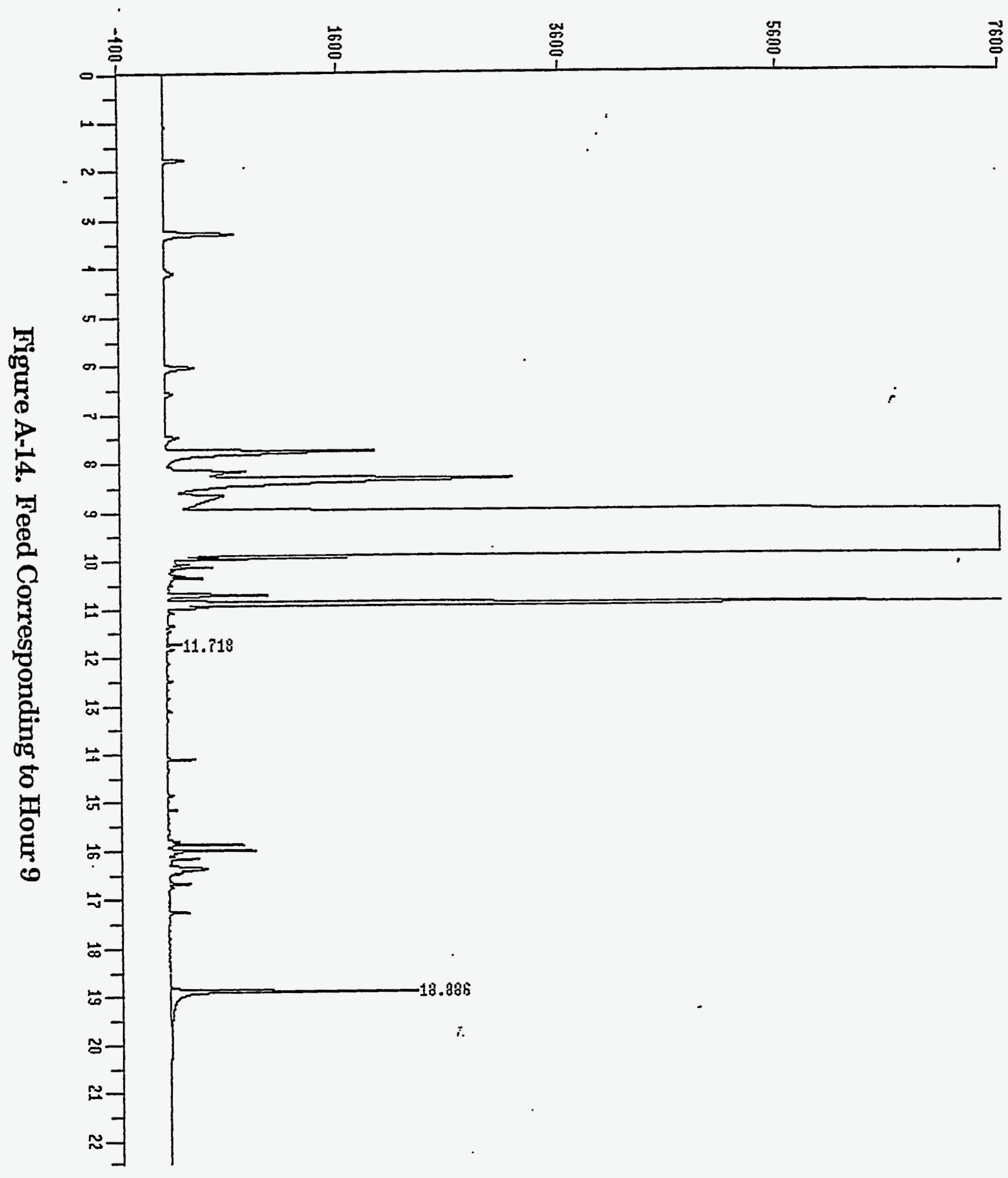

End of plot. Time $\times 0.00$ to 22.51 minutes Chart speed $=0.89 \mathrm{~cm} / \mathrm{min}$ 
Operator:

Method File Name: WebIBENZ.M

Sample Info:

Misc Info:

Integration File Name : DATA:BIBENZ.I

consisting of channels : 1 . GC Signal 2

of BIBENZ.D

Bottle Number : $\theta$ Repetition Number: 1

Calibration file: DATA:BIBENZ.Q Last Update: 19 Jul 95 10:02 am Reference Peak Window: $\quad 5.00$ Absolute Minutes Non-Reference Peak Window: 0.10 absolute Minutes

Sample Amount: 0.000 Uncalibrated Peak RF: 1.000 Multiplier: 1.000

\begin{tabular}{|c|c|c|c|c|c|c|}
\hline & & Ini & Ret & Signal & Compound & \\
\hline Num & Type & Type & Time & $\begin{array}{l}\text { Description } \\
\text { GC Signal } 2\end{array}$ & $\begin{array}{l}\text { Name } \\
\text { BIEENZYL }\end{array}$ & $\begin{array}{ll}\text { Area } & \text { Amount } \\
* * \text { Not Found *** }\end{array}$ \\
\hline 2 & & INU & 18.886 & GC Signel 2 & COMP\#S & 0.32897 WT\% \\
\hline
\end{tabular}

-

Sample peak \#1 not found at retention time $11.840 \div 1-0.050 \mathrm{~min}$.

*** Not all calibrated peaks found *** 
(a) Peng fhrt

$\therefore 0$

$1:$ Sig. 2 of DATA:BIBENZ.D

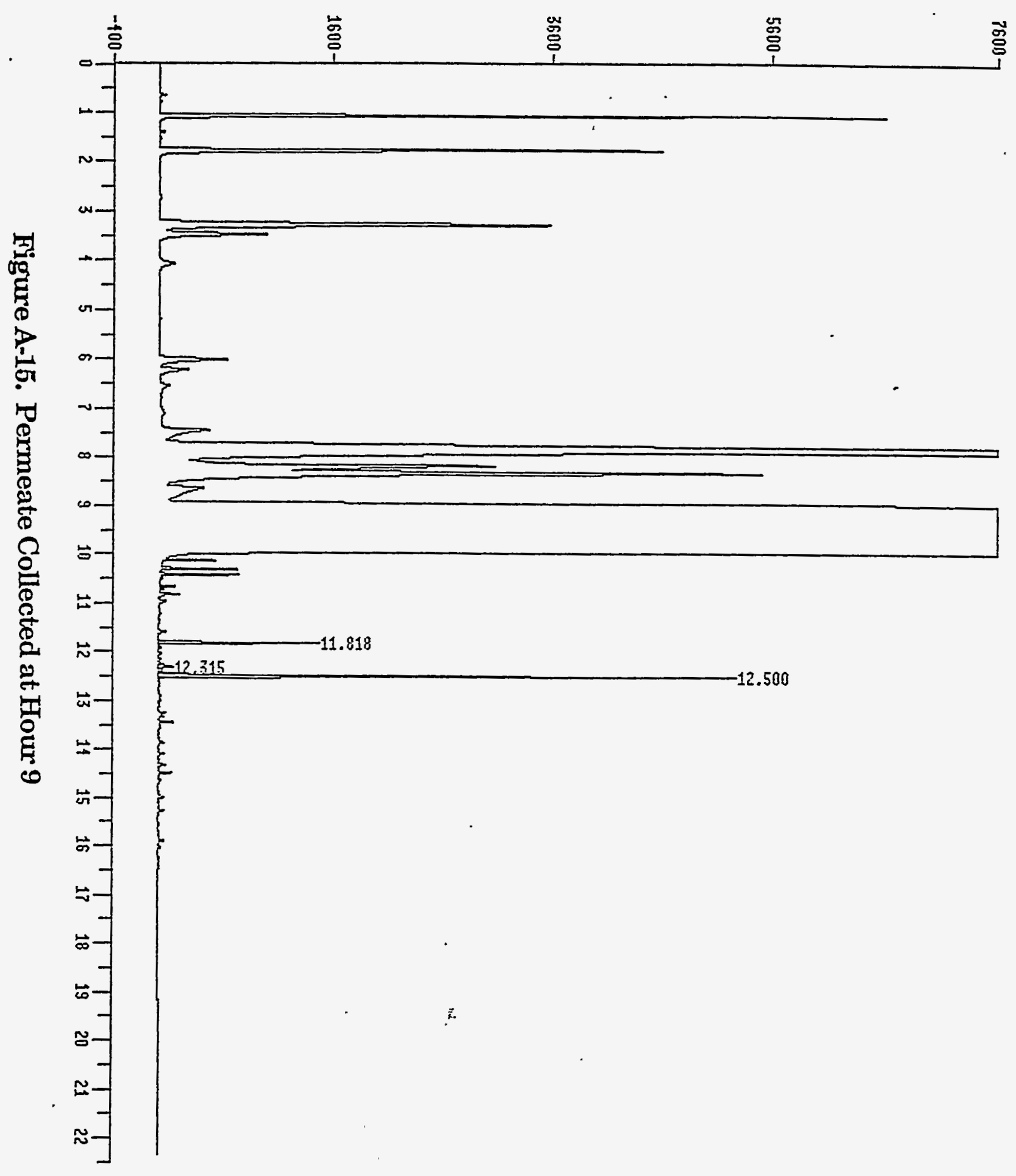

End of plot. Time $=0.00$ to 22.50 minutes Chart speed $=0.89 \mathrm{~cm} / \mathrm{min}$ 
Operator:

Method File Name : WBBIEENz.M

Sample Info:

Misc Info:

Integration File Name : DATA:BIBENZ.I

consisting of channels : 1 . GC Signal $2{ }^{\circ}$ of BIBENZ.D

Botile Number : 0 Repetition Number: 1

Calibration file: DATA:BIBENZ.Q Last Update: 19 Jul 95 10:02 am

$$
\text { Reference Peak Window: } 5.00 \text { Absolute Minutes }
$$

Non-Reference Peak Window: 0.10 Absolute Minutes

Sample Amount: 0.000 Uncalibrated Peak fF: 1.000 Multiplier:.1.000

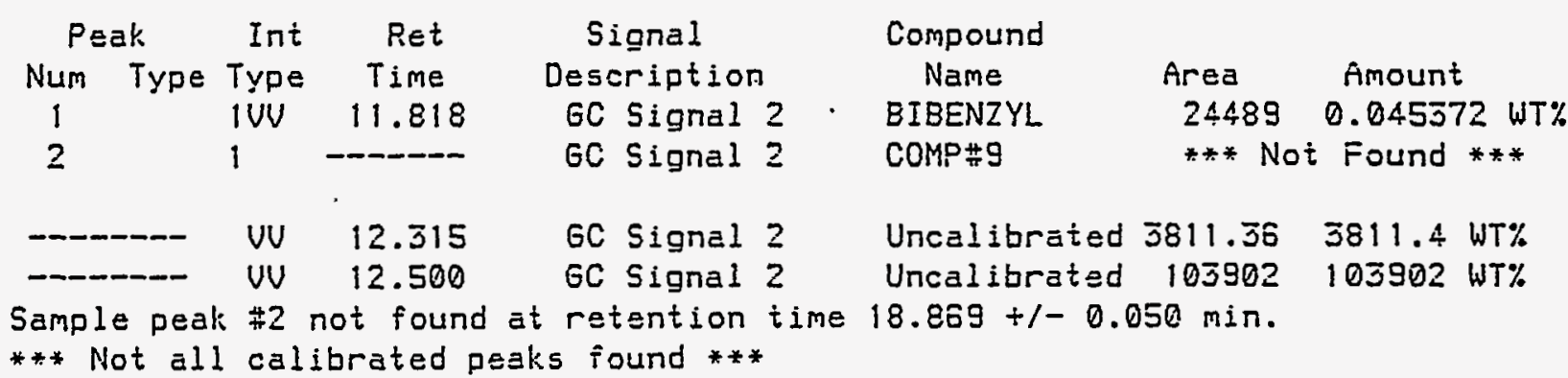


$\begin{array}{rlr}1 & 1 \\ \vdots & & 7-20\end{array}$

1: Sig. 2 of DATA:BIBENZ.D

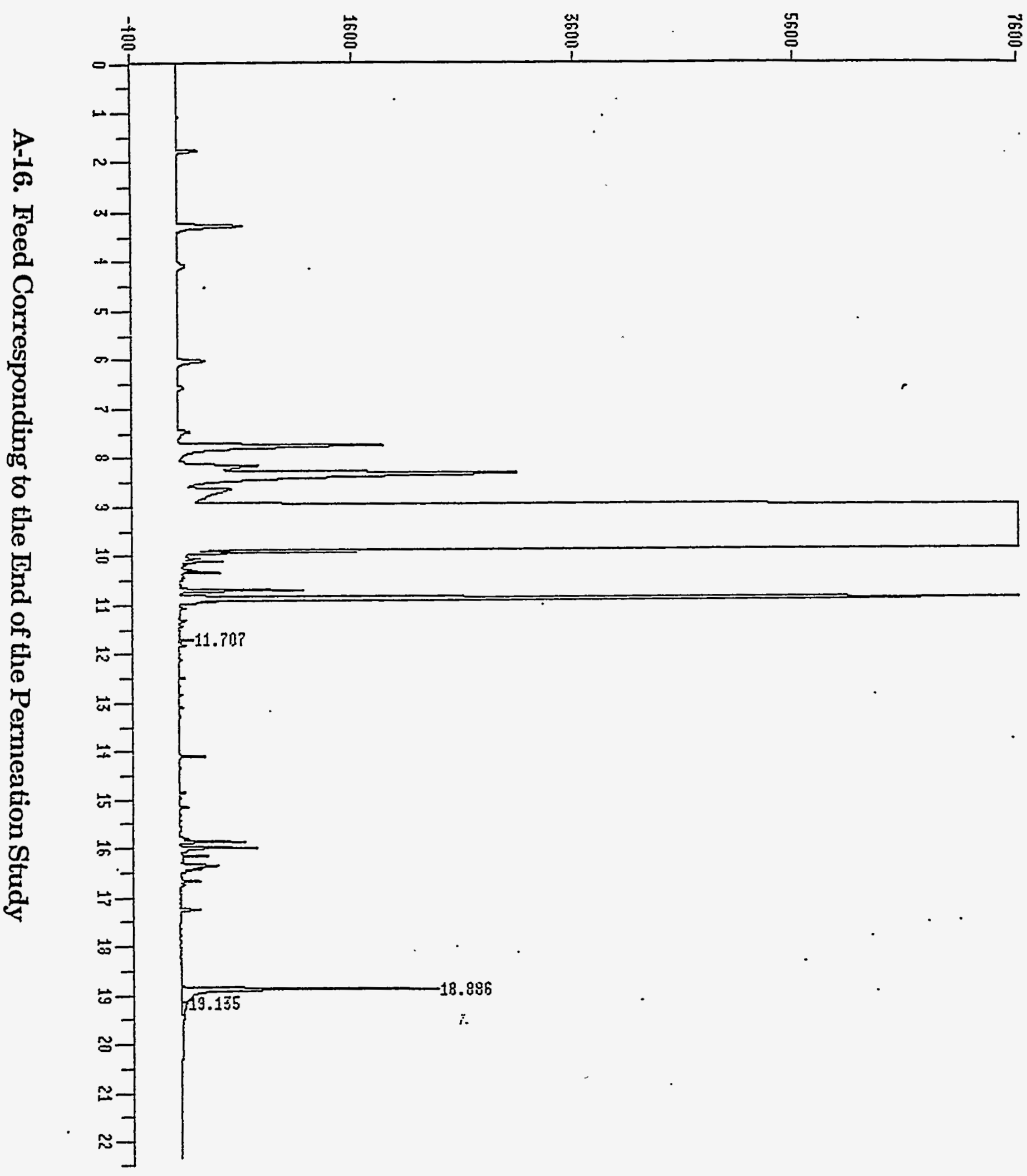

End of plot. Time $=$ 
Operator:

Method File Name : WBBIBENZ.M

21 Jul $958: 51 \mathrm{am}$

Sample Info:

Misc Info:

Integration File Name : DATA:BIBENZ.I

consisting of channels : 1 . GC Signal 2 of BIBENZ.D

Bottle Number : 0 Repetition Number: 1

Calibration file: DATA:BIBENZ.Q Last Update: 19 Jul 95 10:02 am Reierence Peak Window: 5.00 Absolute Minutes Non-Reference Peak Window: 0.10 Absolute Minutes

Sample Amount: 0.000 Uncalibrated Peak RF: 1.000 Multiplier: 1.000

$-7$

\begin{tabular}{|c|c|c|c|c|}
\hline & & Int & Ret & Signal \\
\hline Num & Type & Type & Time & Description \\
\hline 2 & & IUN & 18.884 & GC Signal 2 \\
\hline
\end{tabular}

Compound

Name
BIBENZYL
COMP\#9

Area fimount

*** Not Found ***

$76520 \quad 0.29377$ WT\%

UU 11.700 GC Signal 2 Uncalibrated $2528.55 \quad 2528.7$ WT\%

Sample peak \#1 not found at retention time $11.840+1-0.050 \mathrm{~min}$.

*** Not all calibrated peaks found ***. 


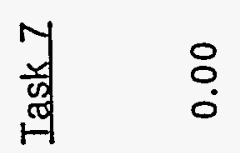

ำ

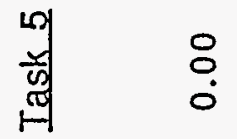

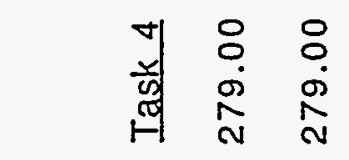

닝

品

(1)

틍 훙

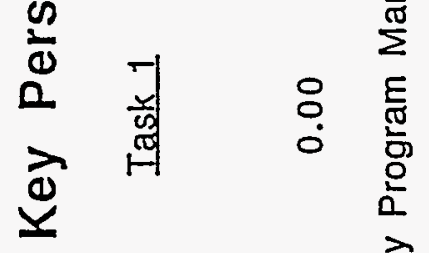

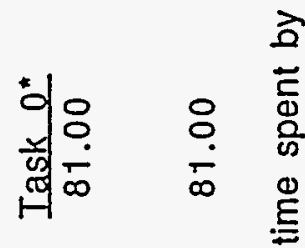

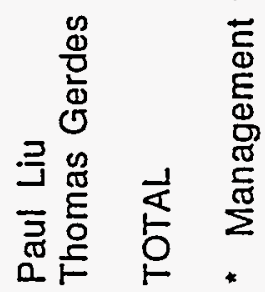

